Preprint typeset in JHEP style - HYPER VERSION

TAUP -2878-08

0805.2799 [hep-ph]

October 30, 2018

\title{
A QCD motivated model for soft interactions at high energies
}

\section{E. Gotsman*, E. Levin`, U. Maor ${ }^{\ddagger}$ and J.S. Miller ${ }^{\S}$}

Department of Particle Physics, School of Physics and Astronomy

Raymond and Beverly Sackler Faculty of Exact Science

Tel Aviv University, Tel Aviv, 69978, Israel

ABSTRACT: In this paper we develop an approach to soft scattering processes at high energies, which is based on two mechanisms: Good-Walker mechanism for low mass diffraction and multi-Pomeron interactions for high mass diffraction. The pricipal idea, that allows us to specify the theory for Pomeron interactions, is that the so called soft processes occur at rather short distances $\left(r^{2} \propto 1 /<p_{t}>^{2} \propto \alpha_{I P}^{\prime} \approx\right.$ $0.01 \mathrm{GeV}^{-2}$ ), where perturbative QCD is valid. The value of the Pomeron slope $\alpha_{I P}^{\prime}$ was obtained from the fit to experimental data. Using this theoretical approach we suggest a model that fits all soft data in the ISR-Tevatron energy range, the total, elastic, single and double diffractive cross sections, including $t$ dependence of the differential elastic cross section, and the mass dependence of single diffraction. In this model we calculate the survival probability of diffractive Higgs production, and obtained a value for this observable, which is smaller than $1 \%$ at the LHC energy range.

Keywords: Soft Pomeron, BFKL Pomeron, Diffractive Cross Sections, Survival Probability

PACS: 13.85.-t, 13.85.Hd, 11.55.-m, 11.55.Bq

\footnotetext{
*Email: gotsman@post.tau.ac.il.

${ }^{\dagger}$ Email: leving@post.tau.ac.il, levin@mail.desy.de.

${ }^{\ddagger}$ Email:maor@post.tau.ac.il.

$\S$ Email:jeremymi@post.tau.ac.il.
} 


\section{Contents}

1. Introduction 2

2. The two channel model and the value of $\alpha_{I P}^{\prime}$ 列

2.1 GLM two channel model 3

$2.2 \alpha_{I P}^{\prime} \longrightarrow 0 \quad$ 送

3. Pomerons Interactions 6

3.1 QCD input 6

3.2 Generating function and a partonic interpretation 8

3.3 Improved Mueller-Patel-Salam-Iancu approximation 11

3.4 High energy amplitude 13

3.5 Diffractive production processes 14

3.6 Our approach 17

4. Results of the fit 18

4.1. Cross sections and elastic slope 18

$4.2 t$ - dependence of the differential elastic cross section 20

1.3. Mass dependence of the diffractive cross section 20

5. Predictions for LHC and Cosmic Rays Energies: $b$-dependence of the amplitudes 21

6. Survival probability of diffractive Higgs production 22

7. Discussion 27 


\section{Introduction}

The goal of this paper is to construct a QCD motivated model for the strong interactions at high energy, and to elucidate the model's predictions and implications at LHC and Cosmic Rays energies.

The difficulties and challenges of a theoretical approach to the strong interaction are well known: a qualitative understanding of the confinement of quarks and gluons in QCD. For high energy scattering the situation is even more difficult than for the hadron structure, since approximate methods such as QCD sum rules and/or effective theories, as well as the lattice QCD approach, cannot be used to calculate the high energy amplitudes. Today, and for the past four decades, the accepted method of describing soft interactions at high energy, is the phenomenology based on the soft Pomeron and secondary Reggeons (see Refs. [1-3] for details). All parameters related to the Pomeron and Reggeons, which are assumed to be simple poles in the J-plane, such as the intercepts and slopes of the trajectories, the vertices and their dependence on the impact parameter, have to be deduced from the experimental data. However, the key problem of all approaches based on the soft Pomeron hypothesis, is that there is no theory which can specify what kind of interactions between Pomerons have to be taken into account, as well as the values of the multi-Pomeron vertices. Due to this problem we are doomed to build basically unreliable models, since the only criteria is that they describe the experimental data (see Refs. [4-7]).

In this paper we attempt to overcome this difficulty by using high energy perturbative QCD approach. At first sight, this appears unrealistic, since the pQCD approach is based on the smallness of the running QCD coupling at short distances, while the high energy interaction is a typical example of long distance non-perturbative QCD. We wish to question this widely held prejudice. Indeed, the only microscopic explanation for the Pomeron structure is given in the partonic approach [8], in which the slope of the Pomeron trajectory is related to the mean transverse momentum of the exchanged partons $\left(\alpha_{I P}^{\prime} \propto 1 /<p_{t}>\right.$, where $\left\langle p_{t}\right\rangle$ is the mean parton momentum). The commonly held view in high energy phenomenology, is that $\alpha_{I P}^{\prime}=0.25 \mathrm{GeV}^{-2}[9]$, which implies that in the Pomeron the typical momenta of partons are high (especially if you compare this value with the value of $\alpha_{I R}^{\prime}=1 \mathrm{GeV}^{-2}$ for the secondary Reggeons). In the next section, we extend our output up to the Planck mass, and will show that the fit to the experimental data based on our model with power-like dependence of the Pomeron-proton vertices on energy, validates the consistency of our calculations with unitarity. The momentum transferred $t$ behaviour leads to $\alpha_{\mathbb{P}}^{\prime}<0.02$ $\mathrm{GeV}^{-2}$. This result, together with the fit of Ref. [7], where the data were fitted with $\alpha_{\mathbb{P}}^{\prime}=0$, lends support to our assumption that, the typical parton momentum is large (approximately $<p_{t}>=1 / \sqrt{\alpha_{I P}^{\prime}} \geq 7 G e V$ ). Therefore, the running QCD coupling $\alpha_{S}=\pi / b \ln \left(<p_{t}^{2}>/ \Lambda_{Q C D}^{2}\right) \ll 1$ (approximately 0.18), and we can consider it as our small parameter, when applying perturbative QCD estimates to the Pomeron-Pomeron interaction vertices.

The theoretical scheme that emerges from such an approach will be discussed in section 3. Using perturbative QCD we select the essential Pomeron-Pomeron vertices, and develop the method for summation of all these diagrams. We also give the partonic interpretation of our approach, and develop an approximate method for the solution of the long standing problem of the high energy asymptotic behaviour of the 
scattering amplitude. Based on this analysis we derive formulae for all cross sections taking into account the Pomeron interactions.

In section 4 we fit the available experimental data using our formalism. This fit is based on an updated data base which includes the published $p-p$ and $\bar{p}-p$ data points of $\sigma_{t o t}$, the integrated values of $\sigma_{e l}, \sigma_{s d}$, $\sigma_{d d}$ and the forward elastic slope $B_{e l}$, in the ISR-Tevatron energy range. $\rho=\frac{\operatorname{Re} a_{e l}(t=0, s)}{\operatorname{Im} a_{e l}(t=0, s)}$ and the forward slope of the SD and DD final states are predictions of the model. The additional data that we have used to validate our present model, are the $t$-dependence of the differential elastic cross section, and the mass dependence of the single diffractive production. We successfully describe this data. Based on this fit we give reliable predictions of the quantities to be measured at the LHC c.m. energy of $14 T e V$. Our output also covers the broad Cosmic Rays energy range up to the GZK limit.

The fifth section is devoted entirely to a calculation of the survival probability $\left(\left\langle\left|S^{2}\right|\right\rangle\right)$ for exclusive central diffractive Higgs production at the LHC, and a discussion on the reliability of these calculations. We confirm the tendency in which $\left\langle\left|S^{2}\right|\right\rangle$ becomes small (less that 1\%), noticed in our previous paper [6]. From a practical point of view, this is the most salient feature of this paper.

In the conclusions, we compare critically our results with other approaches, and summarize our findings. We list a few experimental signatures, which should enable us to differentiate between alternative theoretical options and phenomenological models.

\section{The two channel model and the value of $\alpha_{\mathbb{P}}^{\prime}$}

\subsection{GLM two channel model}

The GLM two channel model has been described in our previous publications (see Refs. $[4,6,10-12]$, and references therein). In this formalism, diffractively produced hadrons at a given vertex are considered as a single hadronic state described by the wave function $\Psi_{D}$, which is orthonormal to the wave function $\Psi_{h}$ of the incoming hadron (proton in the case of interest), $\left\langle\Psi_{h} \mid \Psi_{D}\right\rangle=0$. We introduce two wave functions $\psi_{1}$ and $\psi_{2}$ that diagonalize the $2 \times 2$ interaction matrix $\mathbf{T}$

$$
A_{i, k}=<\psi_{i} \psi_{k}|\mathbf{T}| \psi_{i^{\prime}} \psi_{k^{\prime}}>=A_{i, k} \delta_{i, i^{\prime}} \delta_{k, k^{\prime}}
$$

In this representation the observed states are written in the form

$$
\begin{gathered}
\psi_{h}=\alpha \psi_{1}+\beta \psi_{2}, \\
\psi_{D}=-\beta \psi_{1}+\alpha \psi_{2},
\end{gathered}
$$

where, $\alpha^{2}+\beta^{2}=1$. Using Eq. (2.1), we can rewrite the unitarity constraints in the form

$$
\operatorname{Im} A_{i, k}(s, b)=\left|A_{i, k}(s, b)\right|^{2}+G_{i, k}^{i n}(s, b),
$$

where $G_{i, k}^{i n}$ is the contribution of all non diffractive inelastic processes, i.e. it is the summed probability for these final states to be produced in the scattering of particle $i$ off particle $k$. 
A simple solution to Eq. (2.4) has the same structure as in a single channel formalism,

$$
\begin{gathered}
A_{i, k}(s, b)=i\left(1-\exp \left(-\frac{\Omega_{i, k}(s, b)}{2}\right)\right), \\
G_{i, k}^{i n}(s, b)=1-\exp \left(-\Omega_{i, k}(s, b)\right) .
\end{gathered}
$$

From Eq. (2.6) we deduce, that the probability that the initial projectiles $(i, k)$ reach the final state interaction unchanged, regardless of the initial state rescatterings, is $P_{i, k}^{S}=\exp \left(-\Omega_{i, k}(s, b)\right)$.

For the opacities $\Omega_{i, k}$ we use the expression

$$
\Omega_{i, k}(s, b)=g_{i} g_{k}\left(\frac{s}{s_{0}}\right)^{\Delta_{\mathbb{P}}} S\left(b ; m_{i}, m_{k} ; \alpha_{\mathbb{P}}^{\prime} \ln \left(s / s_{0}\right)\right)
$$

which differs from the expression that we used in our previous models. The profile function $S\left(b, \alpha_{\mathbb{I P}}^{\prime} ; m_{i}, m_{k} ; \ln \left(s / s_{0}\right)\right)$ at $s=s_{0}$, corresponds to the power-like behaviour of the Pomeron-hadron vertices,

$$
\begin{aligned}
& S\left(b ; m_{i}, m_{k} ;, \alpha_{\mathbb{P}}^{\prime} \ln \left(s / s_{0}\right)=0\right)=\int \frac{d^{2} q}{(2 \pi)^{2}} g_{i}(q) g_{k}(q) e^{i \vec{q}_{\perp} \cdot \vec{b}}, \\
& \text { with a normalization } \int d^{2} b S\left(b ; m_{i}, m_{k} ;, \alpha_{\mathbb{P}}^{\prime} \ln \left(s / s_{0}\right)=0\right)=1 .
\end{aligned}
$$

In this paper we choose

$$
g_{i}(q)=\frac{1}{\left(1+q^{2} / m_{i}^{2}\right)^{2}}
$$

The arguments we list in favour of this choice are: (i) pQCD leads to $g_{i}(q) \rightarrow \alpha_{S}^{2}(q) / q^{4}$ [13]; (ii) in some models (for example in the constituent quark model) $g_{i}(q)$ is equal to the electro-magnetic form factor of the proton, which has the form of Eq. (2.9); and (iii) Eq. (2.9) reproduces the experimental elastic differential cross section for $\mathrm{p}-\mathrm{p}(\mathrm{p}-\bar{p})$ data in the range of $t$, up to 1 to $1.5 \mathrm{GeV}^{2}$ [9], while a Gaussian parametrization fits the data only for very small $t \leq 0.1 \mathrm{GeV}^{2}$.

Using Eq. (2.8) we obtain for $S\left(b ; m_{i}, m_{k} ;, \alpha_{\mathbb{P}}^{\prime} \ln \left(s / s_{0}\right)=0\right)$,

$$
\begin{aligned}
& \frac{1}{\left(1+q^{2} / m_{i}^{2}\right)^{2}} \times \frac{1}{\left(1+q^{2} / m_{k}^{2}\right)^{2}} \Longrightarrow S\left(b ; m_{i}, m_{k} ;, \alpha_{\mathbb{P}}^{\prime} \ln \left(s / s_{0}\right)=0\right)= \\
& =\frac{m_{i}^{3} m_{k}^{3}}{4 \pi\left(m_{i}^{2}-m_{k}^{2}\right)^{3}}\left\{4 m_{i} m_{k}\left(K_{0}\left(m_{i} b\right)-K_{0}\left(m_{k} b\right)\right)+\left(m_{i}^{2}-m_{k}^{2}\right) b\left(m_{k} K_{1}\left(m_{i} b\right)+m_{i} K_{1}\left(m_{k} b\right)\right)\right\} .
\end{aligned}
$$

For energies $s>s_{0}$, we need to take into account the observed shrinkage of the diffraction peak. To this end we replace $g_{i}(q) g_{k}(q)$ by $g_{i}(q) g_{k}(q) \exp \left(-\alpha_{\mathbb{P}}^{\prime} \ln \left(s / s_{0}\right) q^{2}\right)$ in Eq. (2.8). To simplify our calculations we replace

$$
m_{i}^{2} \Longrightarrow m_{i}^{2}(s) \equiv \frac{m_{i}^{2}}{1+\alpha_{P}^{\prime} \ln \left(s / s_{0}\right) / 4 m_{i}^{2}}
$$


It is easy to check that $g_{i}\left(q ; m_{i}\right) g_{k}\left(q ; m_{k}\right) \exp \left(-\alpha_{\mathbb{P}}^{\prime} \ln \left(s / s_{0}\right) q^{2}\right)$ and $g_{i}\left(q ; m_{i}(s)\right) g_{k}\left(q ; m_{k}(s)\right)$ have the same behaviour for $\alpha_{\mathbb{P}}^{\prime} \ln \left(s / s_{0}\right) q^{2} \ll 1$. When $\alpha_{\mathbb{P}}^{\prime} \ln \left(s / s_{0}\right) q^{2} \gg 1$ these two expressions are different. Note that the Regge factor $\exp \left(-\alpha_{\mathbb{P}}^{\prime} \ln \left(s / s_{0}\right) q^{2}\right)$ cannot be justified in this kinematic region. In the region of $\alpha_{I P}^{\prime} \ln \left(s / s_{0}\right) q^{2} \sim 1$ it is preferable to use the Regge factor. We assume that $\alpha_{I P}^{\prime}$ is small enough, so that this region gives a negligible contribution to all experimental observables.

In general, we have to consider four possible re-scattering processes in Eq. (2.1). However, in the case of $p-p(\bar{p}-p)$ the two non-diagonal amplitudes are equal $A_{1,2}=A_{2,1}$, and we end up with three rescattering amplitudes. These amplitudes are presented in our two channel formalism in the following form $[4,11,12]$

$$
\begin{gathered}
a_{e l}(s, b)=i\left\{\alpha^{4} A_{1,1}+2 \alpha^{2} \beta^{2} A_{1,2}+\beta^{4} \mathcal{A}_{2,2}\right\}, \\
a_{s d}(s, b)=i \alpha \beta\left\{-\alpha^{2} A_{1,1}+\left(\alpha^{2}-\beta^{2}\right) A_{1,2}+\beta^{2} A_{2,2}\right\}, \\
a_{d d}=i \alpha^{2} \beta^{2}\left\{A_{1,1}-2 A_{1,2}+A_{2,2}\right\} .
\end{gathered}
$$

It should be stressed that in this approach diffraction dissociation, appears as an outcome of the Good and Walker mechanism [14] (G-W) or, in other words, the elastic, single diffraction and double diffraction processes occur due to elastic scatterings of $\psi_{1}$ and $\psi_{2}$, the correct degrees of freedom.

The corresponding cross sections are given by

$$
\begin{aligned}
& \sigma_{t o t}(s)=2 \int d^{2} b a_{e l}(s, b), \\
& \sigma_{e l}(s)=\int d^{2} b\left|a_{e l}(s, b)\right|^{2}, \\
& \sigma_{s d}(s)=\int d^{2} b\left|a_{s d}(s, b)\right|^{2}, \\
& \sigma_{d d}(s)=\int d^{2} b\left|a_{d d}(s, b)\right|^{2} .
\end{aligned}
$$

\section{$2.2 \alpha_{\mathbb{P P}}^{\prime} \longrightarrow 0$}

Using Eq. (2.1) - Eq. (2.7) we fit the experimental data, so as to find the value of $\alpha_{\mathbb{P}}^{\prime}$. However, most of the data are available at rather low energies $W=\sqrt{s}=20$ to $600 \mathrm{GeV}$, where the contributions of the secondary Reggeon have to be included. For this we replace $\Omega_{i, k}(s, b)$ given by Eq. (2.7) by the sum of Pomeron and Reggeon contributions,

$$
\Omega_{i, k}(s, b)=\Omega_{i, k}^{\mathbb{P}}(s, b)\left(\text { see Eq. (2.7)) }+\Omega_{i, k}^{\mathbb{R}}(s, b),\right.
$$

with

$$
\Omega_{i, k}^{\mathbb{R}}(s, b)=\frac{g_{i}^{\mathbb{R}} g_{k}^{\mathbb{R}} \eta\left(\frac{s}{s_{0}}\right)^{\Delta_{\mathbb{R}}}}{\pi R_{i, k}^{2}(s)} \exp \left(-\frac{b^{2}}{R_{i, k}^{2}(s)}\right)
$$




\begin{tabular}{|l|l|l|l|l|l|l|}
\hline$\Delta_{\mathbb{P}}$ & $\beta$ & $\alpha_{\mathbb{P}}^{\prime}$ & $g_{1}$ & $g_{2}$ & $m_{1}$ & $m_{2}$ \\
\hline 0.120 & 0.46 & $0.012 \mathrm{GeV}^{-2}$ & $1.27 \mathrm{GeV}^{-1}$ & $3.33 \mathrm{GeV}^{-1}$ & $0.913 \mathrm{GeV}$ & $0.98 \mathrm{GeV}$ \\
\hline$\Delta_{\mathbb{R}}$ & $\beta$ & $\alpha_{\mathbb{R}}^{\prime}$ & $g_{1}^{\mathbb{R}}$ & $g_{2}^{\mathbb{R}}$ & $R_{0,1}^{2}$ & $\chi^{2} /$ d.o.f. \\
\hline-0.438 & 0.46 & $0.60 \mathrm{GeV}^{-2}$ & $4.0 \mathrm{GeV}^{-1}$ & $118.4 \mathrm{GeV}^{-1}$ & $4.0 \mathrm{GeV}^{-2}$ & 0.87 \\
\hline
\end{tabular}

Table 1: Fitted parameters for two channel (eikonal) model

The signature factor is

$$
\eta=\frac{1 \pm e^{i \pi \alpha_{\mathbb{R}}(q)}}{\sin \left(\pi \alpha_{\mathbb{R}}(q)\right)}
$$

corresponding to a Reggeon trajectory $\alpha_{\mathbb{R}}(q)=1+\Delta_{\mathbb{R}}+\alpha_{\mathbb{R}}^{\prime} q^{2}$, and

$$
R_{i, k}^{2}(s)=R_{0, i}^{2}+R_{0, k}^{2}+4 \alpha_{\mathbb{R}}^{\prime} \ln \left(s / s_{0}\right),=R_{0 ; i, k}^{2}+4 \alpha_{\mathbb{R}}^{\prime} \ln \left(\frac{s}{s_{0}}\right) .
$$

$g_{i}^{R}, R_{0, i}^{2}, \Delta_{\mathbb{R}}$ and $\alpha_{\mathbb{R}}^{\prime}$ are fitted parameters. For the Regge sector we know the natural values for $\Delta_{\mathbb{R}}$ and $\alpha_{\mathbb{R}}^{\prime}$ from the behaviour of the Regge trajectory at $q^{2}<0(t>0)$, since all hadrons lie on these trajectories, $\Delta_{\mathbb{R}} \approx-0.5$ and $\alpha_{\mathbb{R}}^{\prime} \approx 1 \mathrm{GeV}^{-2}$.

Eq. (2.19) - Eq. (2.22) specify our model. In this model we neglect the interactions between Pomerons, but include eikonal type rescatterings. This means that we assume only a G-W origin for all quasi-elastic processes. The fitted parameters are shown in Table 1. The quality of the fit is illustrated in Fig. 1 and Fig. 2, where our fit is shown by dashed lines. The fit is very good with $\chi^{2} /$ d.o.f. $=0.87$. However, this parametrization fails to fully describe the cross sections for single and double diffractive production, yielding values for these cross sections which are approximately two times smaller than the experimental values.

From this fit we have two conclusions: i) we cannot describe the diffractive production in the framework of the G-W mechanism if we assume an exponential parameterization for the proton profile function; and (ii) the value of $\alpha_{I P}^{\prime}$ turns out to be very small $\alpha_{I P}^{\prime}=0.012 \mathrm{GeV}^{-2}$. As we have discussed in the introduction, we conclude from the smallness of $\alpha_{\mathbb{P}}^{\prime}$, that the hard processes which occur at short distances are responsible for the Pomeron structure.

\section{Pomerons Interactions}

\subsection{QCD input}

Our main hypothesis is that the typical distances for a Pomeron exchange are short, and we can use pQCD as a guide for building a theory for Pomeron interactions, based on the small value of $\alpha_{\mathbb{I P}}^{\prime}$, the Pomeron slope that we obtained in the previous section. We recall that the value of $\alpha_{\mathbb{P}}^{\prime}$ in the parton model [8] can be written as

$$
R^{2}(s)=\left\langle b^{2}\right\rangle_{n}=\left\langle\Delta b^{2}\right\rangle n=\frac{4}{\left.<p_{t}^{2}\right\rangle} \rho \ln \left(s / s_{0}\right)=\alpha_{\mathbb{P}}^{\prime} \ln \left(s / s_{0}\right)
$$




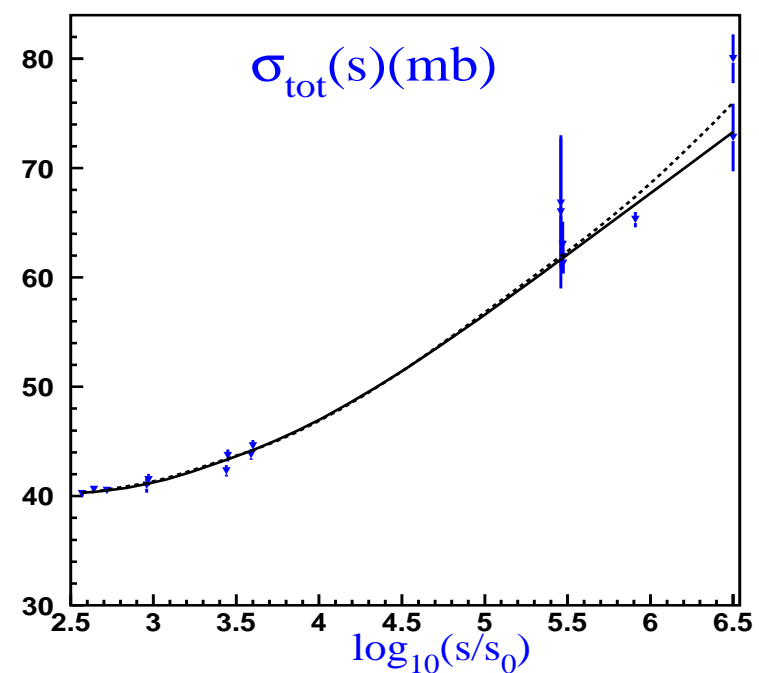

Figure 1: Energy dependence of $\sigma_{\text {tot }}$. The solid line shows the fit with taking into account all Pomeron interactions while the dashed line corresponds to two channel (eikonal) model.

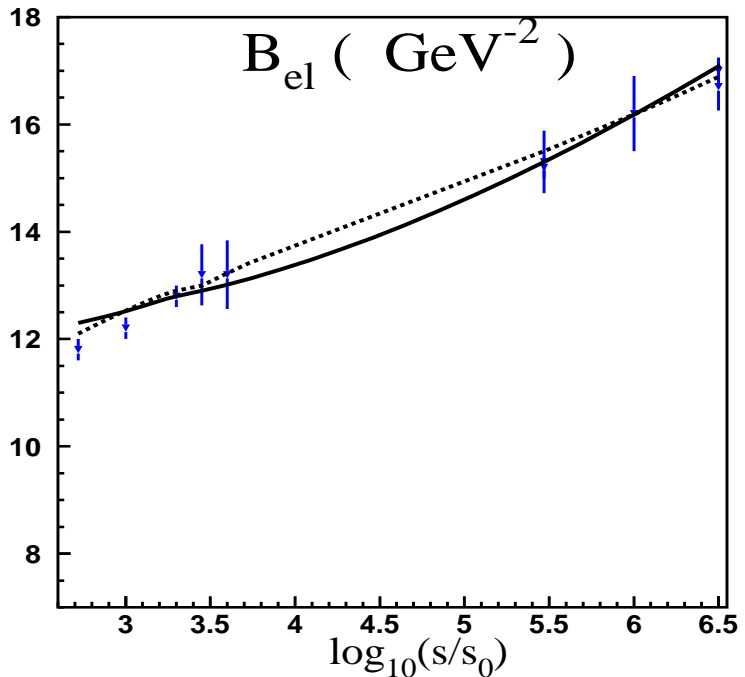

Figure 2: Energy dependence of the slope for the differential elastic cross section. All notation are the same as in Fig. 1

where $n$ is the number of partons at rapidity $Y=\ln \left(s / s_{0}\right)$ and $\rho$ is theb density of partons in a unit of rapidity. In Eq. (3.1) the radius of interaction, is determined as the average distance in impact parameter space that a 'wee' parton can reach at given enegy.

In Eq. (3.1) $R^{2}(s)$ is the radius of interaction at energy $W=\sqrt{s}$, which is the distance in impact parameter $b$ space, that is reached by partons in a two dimensional diffusion, after $n$ steps. $\left\langle\Delta b^{2}\right\rangle$ is the mean displacement during one diffusion step, which can be estimated using the uncertainty principle as $\left\langle\Delta b^{2}\right\rangle=4 /\left\langle p_{t}^{2}\right\rangle$. $\left\langle p_{t}\right\rangle$ is the average parton transverse momentum which we wish to evaluate. The number of diffusion steps is equal to the number of partons, since at each step one parton is emitted. At a given energy the average number of emitted partons is equal to $\rho \ln \left(s / s_{0}\right)$, where $\rho$ is the parton density in units of rapidity. Comparing $n$ with the hadron multiplicities, we conclude that $\rho \approx 1$ or larger. Therefore, Eq. (3.1) leads to $<p_{t}^{2} \geq 20 \mathrm{GeV}$ for $\alpha_{\mathbb{P}}^{\prime}=0.01 \mathrm{GeV}^{-2}$. The typical value of the running QCD coupling, which corresponds to this value of the parton momentum, is $\alpha_{S} \approx 0.15$, which is small enough to be used in pQCD estimates.

In our procedure we only use general results of the perturbative QCD approach to high energy scattering [15]. Consequently:

1.) In the leading order approximation of $\mathrm{pQCD}$, only a Pomeron splitting into two Pomerons and two Pomeron merging into one Pomeron, should be taken into account [16,17], while all other vertices are small. Therefore, using this input from pQCD, we restrict ourselves by summing Pomeron diagrams with triple Pomeron vertices only. 
2) Since $4 \alpha_{I P}^{\prime} \ln (s) \ll 1$ over the entire kninematical range, we have investigated, we can neglect the value of $\alpha_{\mathbb{P}}^{\prime}$, and consider the theory with $\alpha_{\mathbb{P}}^{\prime}=0$.

3) We obtain the anticipated values for all ingredients of the Pomeron interaction approach: the intercept of the Pomeron $\Delta_{\mathbb{P}}$ above unity $\propto \alpha_{S}$, and the triple Pomeron vertex coupling $g_{3 \mathbb{P}} \propto \alpha_{S}^{2}$.

The theory that includes all the above ingredients can be formulated in functional integral form [17],

$$
Z\left[\Phi, \Phi^{+}\right]=\int D \Phi D \Phi^{+} e^{S} \text { with } S=S_{0}+S_{I}+S_{E}
$$

where $S_{0}$ describes free Pomerons, $S_{I}$ corresponds to their mutual interaction and $S_{E}$ relates to the interaction with the external sources (target and projectile). Since $\alpha_{\mathbb{P}}^{\prime}=0, S_{0}$ has the form

$$
S_{0}=\int d Y \Phi^{+}(Y)\left\{-\frac{d}{d Y}+\Delta\right\} \Phi(Y) .
$$

$S_{I}$ includes only triple Pomeron interactions and has the form

$$
S_{I}=g_{3 \mathbb{P}} \int d Y\left\{\Phi(Y) \Phi^{+}(Y) \Phi^{+}(Y)+\text { h.c. }\right\} .
$$

For $S_{E}$ we have local interactions both in rapidity and in impact parameter space,

$$
S_{E}=-\int d Y \sum_{i=1}^{2}\left\{\Phi(Y) g_{i}(b)+\Phi^{+}(Y) g_{i}(b)\right\},
$$

where $g_{i}(b)$ stands for the interaction vertex with the hadrons at fixed $b$.

In the next sections, after specifying this theory, we solve it. Indeed, this theory as any theory of Pomeron interactions, is written in such a way that an high energy amplitude satisfies $t$-channel unitarity. However, $s$-channel unitarity remains a problem. In the next subsection we will change the interaction term $\left(S_{I}\right)$, in such a way, that the theory will have a clear partonic interpretation, and will, also, satisfy $s$-channel unitarity.

\subsection{Generating function and a partonic interpretation}

To find a reformulation given by the functional of Eq. (3.2), we consider a system of partons* that can decay and merge: one parton to two partons, and two partons into one parton, with probabilities $\Gamma(1 \rightarrow 2)$ and $\Gamma(2 \rightarrow 1)$, respectively. For such a system of partons, we can write a simple equation. Indeed, let $P_{n}(y)$ be the probability to find $n$-parton (dipoles) with rapidity $y$ in the wave function of the fastest (parent) parton (dipole), moving with rapidity $Y>y$. For $P_{n}(y)$, we can easily write down a recurrence equation (see Refs. [20,21])

$$
-\frac{\partial P_{n}(y)}{\partial y}=\Gamma(1 \rightarrow 2)\left\{-n P_{n}+(n-1) P_{n-1}\right\}+\Gamma(2 \rightarrow 1)\left\{-n(n-1) P_{n}+(n+1) n P_{n+1}\right\} .
$$

${ }^{*}$ The partons for high energy QCD are the colourless dipoles, as was shown in Ref. [18]. 
In each bracket the first term on the r.h.s., can be viewed as a probability of a dipole annihilation in the rapidity range $(y$ to $y-d y)$ (death term). The second is a probability to create one extra dipole (birth term). Note the negative sign in front of $\partial P_{n}(y) / \partial y$. It appears due to our choice of the rapidity evolution which starts at the largest rapidity $y=Y$, of the fastest dipole and then decreases. The first two terms are responsible for the process of parton decay, while the last two terms describe the contribution of partons merging.

It is useful to introduce the generating function $[18,19,21]$

$$
Z(y, u)=\sum_{n} P_{n}(y) u^{n}
$$

At rapidity $y=Y$ there is only one fastest parton (dipole), which is $P_{1}(y=Y)=1$, while $P_{n>1}(y=$ $Y)=0$. This is the initial condition for the generating function

$$
Z(y=Y)=u \text {. }
$$

At $u=1$

$$
Z(y, u=1)=1
$$

which follows from the physical meaning of $P_{n}$ as a probability.

Eq. (3.6) can be rewritten as an equation in partial derivatives for the generating function $Z(y, u)$,

$$
-\frac{\partial Z(y, u)}{\partial y}=-\Gamma(1 \rightarrow 2) u(1-u) \frac{\partial Z(y, u)}{\partial u}+\Gamma(2 \rightarrow 1) u(1-u) \frac{\partial^{2} Z(y, u)}{\partial^{2} u} .
$$

The description of the parton system given by Eq. (3.10), is equivalent to the path integral of Eq. (3.2). Indeed, the general solution of Eq. (3.10) has a form

$$
Z(Y ; u)=e^{H(u)\left(Y-Y_{0}\right)} Z\left(Y_{0} ; u\right)
$$

with the operator $H$ defined as

$$
H(u)=-\Gamma(1 \rightarrow 2) u(1-u) \frac{\partial}{\partial u}+\Gamma(2 \rightarrow 1) u(1-u) \frac{\partial^{2}}{(\partial u)^{2}}
$$

and

$$
Z\left(Y_{0} ; u\right)=e^{g(b)(u-1)} .
$$

We introduce operators of creation $\left(a^{+}\right)$and annihilation $(a)$ that satisfy $\left[\hat{a}, \hat{a}^{+}\right]=1$, at fixed Y.

$$
\hat{a}=\frac{\partial}{\partial u}, \hat{a}^{+}=u
$$

In this formalism

$$
H=-\Gamma(1 \rightarrow 2) \hat{a}^{+}\left(1-\hat{a}^{+}\right) \hat{a}+\Gamma(2 \rightarrow 1) \hat{a}^{+}\left(1-\hat{a}^{+}\right) \hat{a}^{2},
$$


and the initial state at $Y=Y_{0}$ is defined as

$$
\left|Y_{0}>=e^{g(b)\left(\hat{a}^{+}-1\right)}\right| 0>,
$$

with the vacuum defined as $\hat{a} \mid 0>=0$.

The theory with the Hamiltonian of Eq. (3.15), has an equivalent description using the path integral of Eq. (3.2) (see the detailed derivation in Ref. [24]) with

$$
\begin{aligned}
S & =\int\left(\Phi^{+} \frac{d}{d Y} \Phi+H\left(\Phi^{+}+1,-\Phi\right)\right) d Y=\int d Y\left(\Phi^{+} \frac{d}{d Y} \Phi\right. \\
& \left.-\Gamma(1 \rightarrow 2) \Phi^{+} \Phi+\Gamma(1 \rightarrow 2) \Phi^{+}(\Phi)^{2}+\Gamma(2 \rightarrow 1)\left(\Phi^{+}\right)^{2} \Phi-\Gamma(2 \rightarrow 1)\left(\Phi^{+}\right)^{2}(\Phi)^{2}\right) .
\end{aligned}
$$

Comparing Eq. (3.17) with Eq. (3.3) - Eq. (3.5), one can see that they are similar if we put $\Gamma(1 \rightarrow 2)=\Delta$ and $\Gamma(2 \rightarrow 1)=g_{3 \mathbb{P}}^{2} / \Delta$. However, Eq. (3.17) has an additional term $\left(-\Gamma(2 \rightarrow 1)\left(\Phi^{+}\right)^{2}(\Phi)^{2}\right)$ which describes the two Pomeron to two Pomeron transition (four Pomeron interaction). This term ensures that our approach satisfies $s$-channel unitarity (see Ref. [25]), where it is shown that without this term there is no probabilistic interpretation of the Pomeron interaction theory, and $s$-channel unitarity is violated. We also need to renormalize $\Phi^{+} \rightarrow\left(g_{3 \mathbb{P}} / \Delta\right) \Phi^{+}$and $\Phi \rightarrow\left(\Delta / g_{3 \mathbb{P}}\right) \Phi$.

Using the functional $Z$, we find the scattering amplitude [22], using the following formula:

$$
N(Y) \equiv \operatorname{Im}_{e l}(Y)=\left.\sum_{n=1}^{\infty} \frac{(-1)^{n}}{n !} \frac{\partial^{n} Z(y, u)}{\partial^{n} u}\right|_{u=1} \gamma_{n}\left(Y=Y_{0}, b\right)
$$

where $\gamma_{n}\left(Y=Y_{0}, b\right)$ is the scattering amplitude of $n$-partons (dipoles) at low energy. These amplitudes depend on the impact parameters which are the same for all $n$ partons, since $\alpha_{I P}^{\prime}=0$, and we neglect the diffusion of partons in impact parameter space. Eq. (3.18) corresponds to the partonic approach $[8,23]$, in which a high energy scattering can be viewed as a two stage process. The first stage is a development of the partonic wave function, which we consider by introducing the generating function $Z$. In Eq. (3.18), the second stage is the interaction of the lowest energy partons ('wee' partons) with the target, which is described by the amplitudes $\gamma_{n}\left(Y=Y_{0}, b\right)$. Assuming that there are no correlations between the interacting partons (dipoles) at low energy, we can consider $\gamma_{n}\left(Y=Y_{0}, b\right)=\gamma_{1}^{n}\left(Y=Y_{0}, b\right)$ [22], which we include, choosing $S_{E}$ in the form of Eq. (3.5) and of Eq. (3.16).

The generating function approach given by Eq. (3.7), Eq. (3.10) and Eq. (3.18), has the advantage that it can be solved analytically (see Ref. [26]). This solution leads to a constant cross section at high energy, while the interaction without the four Pomeron term, decreases at high energy [27]. This fact emphasizes the importance of $s$-channel unitarity, in finding the asymptotic behaviour of the scattering amplitude at high energy. Having an exact solution we are able to develop approximate methods, which we can check against the exact solution. 


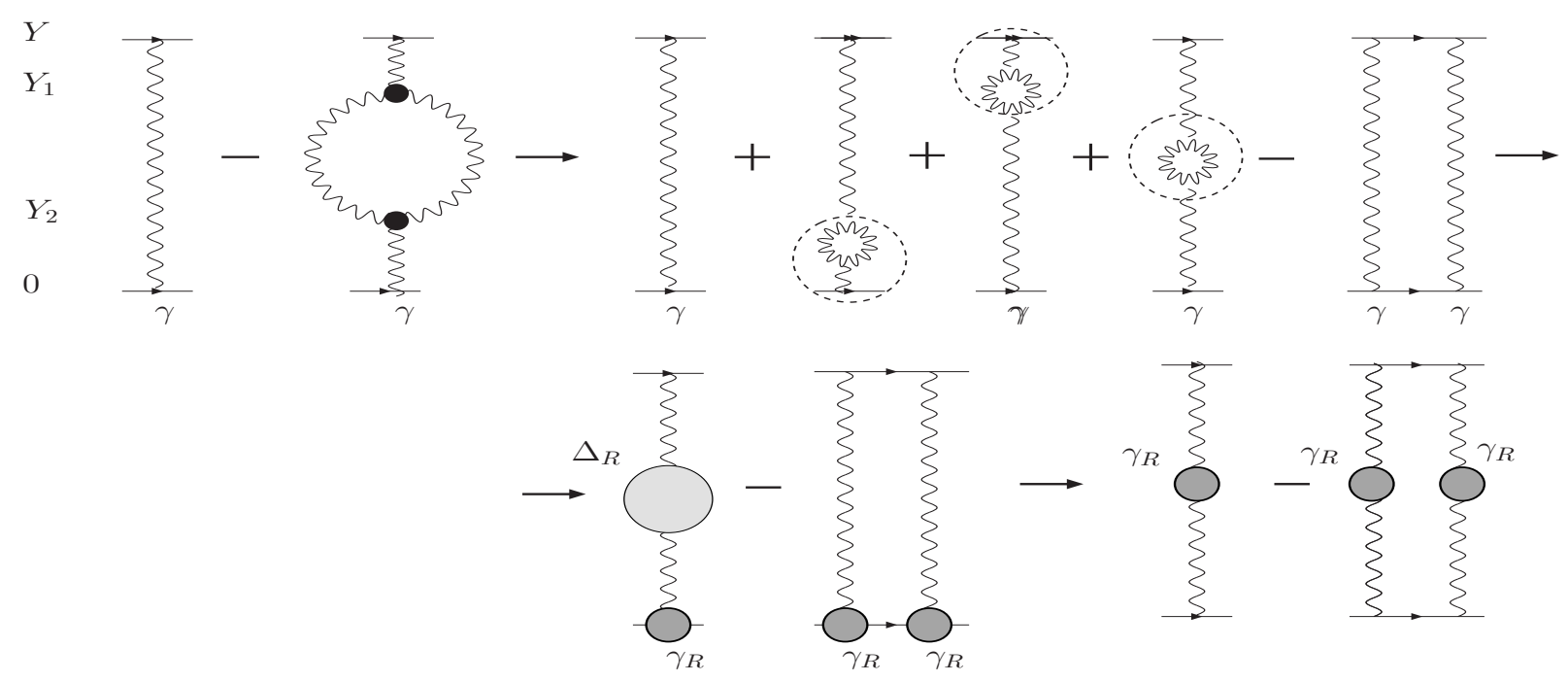

Figure 3: The different contribution to the first enhanced diagram and its renormalization procedure .

\subsection{Improved Mueller-Patel-Salam-Iancu approximation}

Calculating the high energy amplitude in the generating function approach, we use the approximation which allows us to write a simple analytical formulae for the physical observable. The main idea of this approximation, which we call the improved Mueller-Patel-Salam-Iancu approximation, is the following: we claim that at high energy, in the kinematical region

$$
Y \leq \frac{\Delta_{I P}^{2}}{g_{3 \mathbb{P}}^{2}} \equiv \frac{1}{\gamma}
$$

only large Pomeron loops, with a rapidity size of the order of $Y$, contribute to the high energy asymptotic behaviour of the scattering amplitudes. This approximation has been discussed in detail in Refs. [28-30], and here we illustrate this idea using the example of the first Pomeron loop diagram given in Fig. 3. Using Eq. (3.2) or the generating function approach, we obtain the contribution of this diagram in the form ${ }^{\dagger}$

\footnotetext{
${ }^{\dagger}$ In Eq. 3.20 we redefine the hadron-Pomeron vertices denoting them by $g(b) \sqrt{\gamma}$. We will clarify our reason for doing so below. For simplicity we use $g(b)$ for this vertex, neglecting the different hadronic states in our two channel model.
} 


$$
\begin{aligned}
& A(\text { Fig. 3) }= \\
& =g^{2}(b) \gamma G(Y-0)-g^{2}(b) \Gamma(1 \rightarrow 2) \Gamma(2 \rightarrow 1) \gamma \int_{0}^{Y} d y_{1} \int_{0}^{y_{1}} d y_{2} G\left(Y-y_{1}\right) G^{2}\left(y_{1}-y_{2}\right) G\left(y_{2}-0\right) \\
& =g^{2}(b) \gamma G(Y-0)-g^{2}(b) \Gamma(1 \rightarrow 2) \Gamma(2 \rightarrow 1) \gamma \int_{0}^{Y} d y_{1} \int_{0}^{y_{1}} d y_{2} e^{\Delta_{\mathbb{P}}\left(Y+y_{1}-y_{2}\right)} \\
& =g^{2}(b) \gamma e^{\Delta_{-} \mathbb{P} Y}-g^{2}(b) \Gamma(1 \rightarrow 2) \Gamma(2 \rightarrow 1) \gamma\left(\frac{1}{\Delta_{\mathbb{P}}^{2}} e^{2 \Delta_{\mathbb{P}} Y}-\frac{1}{\Delta_{\mathbb{P}}^{2}} e^{\Delta_{\mathbb{P}} Y}-\frac{Y}{\Delta_{p} o m} e^{\Delta_{\mathbb{P}} Y}\right) \\
& =g^{2}(b)\left\{\gamma e^{\Delta_{\mathbb{P}} Y}-\gamma^{2} e^{2 \Delta_{\mathbb{P}} Y}+\gamma^{2} e^{\Delta_{\mathbb{P}} Y}+\gamma(\Gamma(2 \rightarrow 1) Y) e^{\Delta_{p} o m}\right\} \\
& \longrightarrow g^{2}(b)\left\{\gamma_{R} e^{\Delta_{R} Y}-\gamma_{R}^{2} e^{2 \Delta_{\mathbb{P}} Y}\right\},
\end{aligned}
$$

with $\gamma_{R}=\gamma+\gamma^{2}$, and the renormalized intercept of the Pomeron $\Delta_{R E}=\Delta_{\mathbb{P}}+\Gamma(2 \rightarrow 1)=\Delta_{\mathbb{P}}+\Delta_{\mathbb{P}} \gamma$. In the kinematic region of Eq. (3.19) we can neglect the renormalization of the intercept, since in this region $\Delta_{\mathbb{P}} \gamma Y \ll 1$

The general procedure for summing large Pomeron loops was suggested by Mueller, Patel, Salam and Iancu (MPSI) in Ref. [28]. In this approach, the scattering amplitude is calculated using the unitarity constraints in the $t$-channel (assuming that the amplitudes at high energy are purely imaginary, namely $N=\operatorname{Im} A)$,

$$
N([\ldots] \mid Y)=N\left([\ldots] \mid Y-Y^{\prime} ; P \rightarrow n P\right) \bigotimes N\left([\ldots] \mid Y^{\prime} ; P \rightarrow n P\right) .
$$

$\bigotimes$ stands for all necessary integrations, while [...] describes all quantum numbers. The amplitude on the LHS of Eq. (3.22), describes all the enhanced diagrams, while the amplitude on the RHS of this equation, corresponds to the splitting of one Pomeron to $n$ Pomerons. The precise meaning of this equation, will become clear in the next equation. The convenient form of Eq. (3.22), has been written [20,26,28] in terms of the generating functional of Eq. (3.7), and it takes the form

$$
N(Y)=\left.\left.\sum_{n=1}^{\infty} \frac{(-1)^{n}}{n !} \gamma^{n} \frac{\partial^{n} Z^{p}\left(Y-Y^{\prime}, u_{p}\right)}{\partial^{n} u_{p}}\right|_{u_{p}=1} \frac{\partial^{n} Z^{t}\left(Y^{\prime}, u_{t}\right)}{\partial^{n} u_{t}}\right|_{u_{t}=1} .
$$

In Eq. (3.23) we denote by $Z^{p}$ and $Z^{t}$ the generating functions that describe projectile and target respectively.

Eq. (3.23) shows that each dipole with rapidity $Y^{\prime}$ from the target, can interact with any dipole from the projectile, (see Fig. 目) with the scattering amplitude $\gamma$. The factor $1 / n$ ! in Eq. (3.23) appears due to the identity of Pomerons. Eq. (3.23) is defined in the kinematic region of Eq. (3.19), and has a clear physical meaning, being the scattering amplitude of two partons (two dipoles) at low energy $Y_{0} \leq 1 / \Delta_{\mathbb{P}}$. Eq. (3.23) gives a natural generalization of Eq. (3.18), with an obvious physical interpretation, that it is the sum of terms, and each of these terms is the product of probabilities to find $n$-dipoles in the projectile and target, multiplied by the scattering amplitude. Since we are discussing the generating functional that 


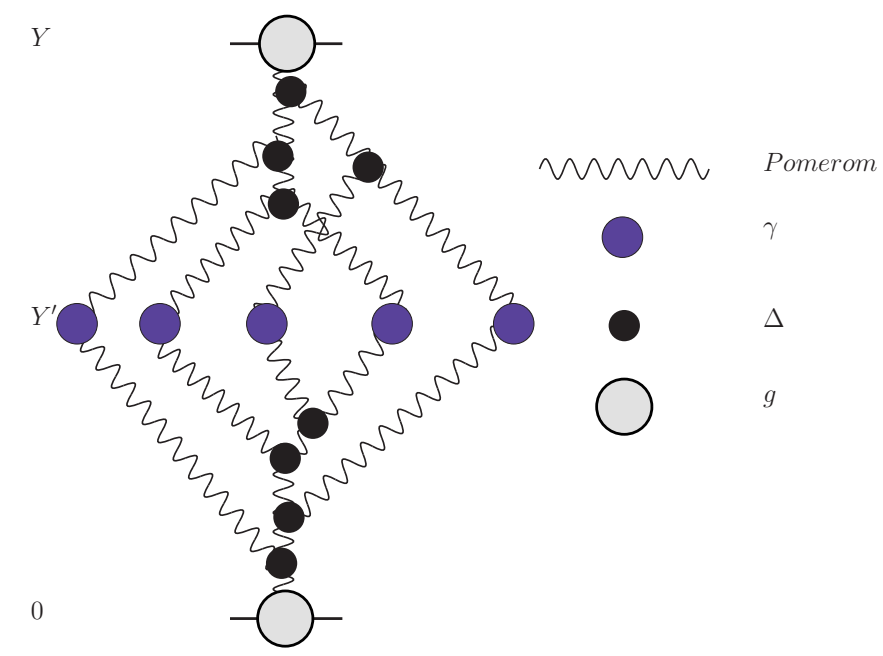

Figure 4: An example of enhanced diagrams, that contribute to the unitarity constraint in the $t$-channel. Wave lines denote the Pomerons. $\gamma$ is the amplitude of the dipole-dipole interaction at low energies (at rapidity $\left.Y_{0} \approx 1 / \bar{\alpha}_{S}\right)$. The particular set of diagrams shown in this figure, corresponds to the MPSI approach $[26,28]$.

satisfies Eq. (3.8) as the initial condition, Eq. (3.23) gives the sum of enhanced diagrams or, in other words, at high energy it leads to a new resulting Green's function of the Pomeron.

The generating functions for the projectile $Z^{p}\left(Y-Y^{\prime}\right)$ and for the target $Z^{t}\left(Y^{\prime}\right)$ in Eq. (3.23), satisfy a very simple equation that describes the parton cascades, in which a parton can only decay into two partons. This equation has the form

$$
-\frac{\partial Z(y, u)}{\partial y}=-\Delta_{\mathbb{P}} u(1-u) \frac{\partial Z(y, u)}{\partial u} .
$$

The above equation has the solution

$$
Z(y, u)=\frac{u}{u+(1-u) e^{\Delta_{\mathbb{P}} y}}=\frac{1}{1+\gamma_{R} e^{\Delta_{\mathbb{P}} y}},
$$

where $u=1 /\left(1+\gamma_{R}\right)$. Eq. (3.25) satisfies the initial and boundary conditions of Eq. (3.8) and Eq. (3.9). Using Eq. (3.25) and Eq. (3.18) it is easy to show that the amplitude is equal to

$$
N(Y)=1-Z\left(u=1 / \gamma_{R}\right)=\sum_{n=1}(-1)^{n} \gamma_{R}^{n} e^{n \Delta_{\mathbb{P}} Y} .
$$

Eq. (3.26) sums the 'fan' Pomeron diagrams and corresponds to the mean field approximation (MFA) of our problem.

\subsection{High energy amplitude}

Using MFA and Eq. (3.26), we can rewrite Eq. (3.23) as

$$
\begin{aligned}
N_{e l}^{M P S I}(Y) & =\left.\left.\sum_{n=1}^{\infty}(-1)^{n+1} \frac{1}{n !} \gamma^{n} \frac{\partial^{n} N^{M F A}\left(Y-Y^{\prime}, \gamma_{R}^{p}\right)}{\partial^{n} \gamma_{R}^{p}}\right|_{\gamma_{R}^{p}=0} \frac{\partial^{n} N^{M F A}\left(Y^{\prime}, \gamma_{R}^{t}\right)}{\partial^{n} \gamma_{R}^{t}}\right|_{\gamma_{R}^{t}=0} \\
& =1-\left.\left\{\exp \left(-\gamma \frac{\partial}{\partial \gamma_{R}^{p}} \frac{\partial}{\partial \gamma_{R}^{t}}\right) N^{M F A}\left(Y-Y^{\prime}, \gamma_{R}^{p}\right) N_{0}^{M F A}\left(Y^{\prime}, \gamma_{R}^{t}\right)\right\}\right|_{\gamma_{R}^{p}=0 ; \gamma_{R}^{t}=0} .
\end{aligned}
$$


Substituting Eq. (3.25) into Eq. (3.27), we obtain that [33,34]

$$
N_{e l}^{M P S I}(Y)=1-\exp \left(\frac{1}{T(Y)}\right) \frac{1}{T(Y)} \Gamma\left(0, \frac{1}{T(Y)}\right)
$$

where $\Gamma(0, x)$ is the incomplete gamma function (see formsph1.epsulae $\mathbf{8 . 3 5 0} \mathbf{- 8 . 3 5 9}$ in Ref. [36]) and

$$
T(Y)=\gamma e^{\Delta_{\mathbb{P}} Y}
$$

In Ref. [34], the solution given by Eq. (3.28), is compared with the exact solution (see Fig.13 of Ref. [34]). It turns out to within a $5 \%$ accuracy, that the MPSI approximation describes the high energy behaviour of the amplitude. At high energy Eq. (3.28) gives $N_{e l}^{M P S I}(Y) \rightarrow 1$.

As has been mentioned, since Eq. (3.23) satisfies the initial condition of Eq. (3.8), it describes, as well, Eq. (3.28), the set of enhanced diagrams, and gives the resulting Pomeron Green's function

$$
G_{\mathbb{P}}^{M P S I}\left(Y-Y^{\prime}\right)=N_{e l}^{M P S I}\left(Y-Y^{\prime}\right)
$$

Replacing the bare Pomeron Green's function by Eq. (3.30), we can use Eq. (2.12), Eq. (2.15) and Eq. (2.16), to calculate elastic and total cross sections.

\subsection{Diffractive production processes}

The conclusion derived from the previous discussion, is that we cannot use the formulae of Eq. (2.13) and Eq. (2.14), for calculating the single and double diffraction cross sections. Indeed, these two equations describe the diffraction production due to the G-W mechanism, while the sum of all enhanced diagrams leads to a new source of diffractive production (see Fig. 5 for examples of such processes).

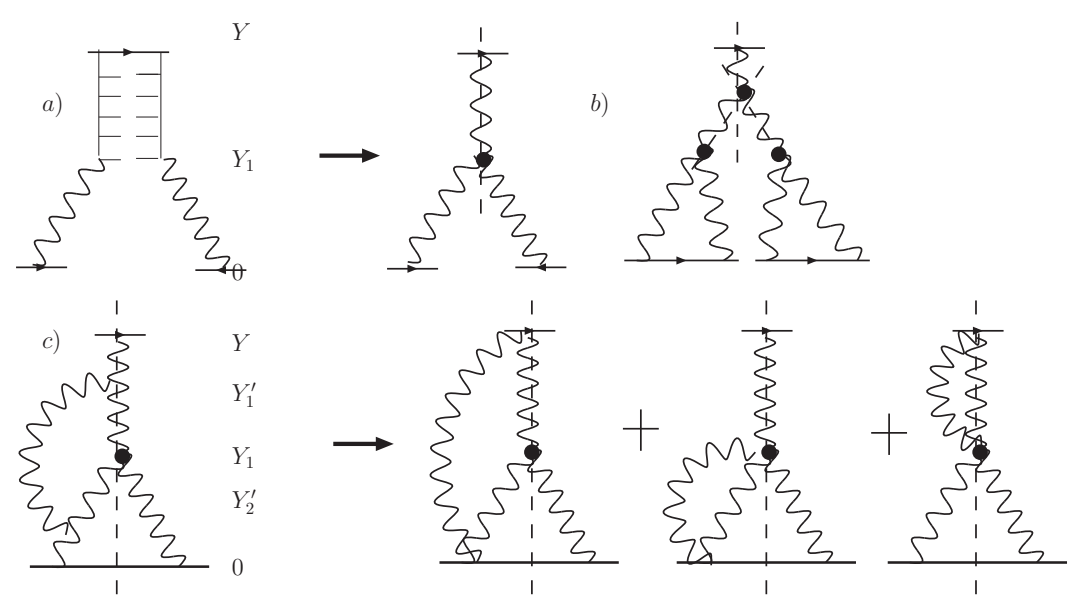

Figure 5: Several examples of the Pomeron diagrams that lead to a different source of the diffractive dissociation that cannot be described in the framework of the G-W mechanism. Fig. 5 -a is the simplest diagram that describes the process of diffraction in the region of large mass $Y-Y_{1}=\ln \left(M^{2} / s_{0}\right)$. Fig. 周 $\mathrm{b}$ and Fig. F g give examples of more complicated diagrams in the region ofsph1.eps large mass. The dashed line shows the cut Pomeron, which describes the production of hadrons (see Fig. 目-a which illustrates this point).

We use the MPSI approximation to obtain the expression for the additional contribution to G-W mechanism. The main idea is shown in Fig. 6. As was discussed in Ref. [29] , in order to apply the MPSI 
approach to diffractive production, we need to consider the generating function of three variables: $w, \bar{w}$ and $v_{\text {in }}$

$$
Z\left(w, \bar{w} ; v_{i n} ; Y\right),=\sum_{n=0 ; m=0 ; k=0}^{\infty} P(n, n, k \mid Y) w^{n} \bar{w}^{m} v_{i n}^{k}
$$

$P(n, n, k \mid Y)$ is the probability to find $n$ and $m$ Pomerons in the amplitude and conjugated amplitude respectively, while $k$ is the number of cut Pomerons (see Ref. [37]). $Z\left(w, \bar{w} ; v_{i n} ; Y\right)$ has been found in Ref. [29] for the parton cascade, with a decay of one parton to two partons,

$$
\begin{array}{ll}
\text { sph1.eps } & Z\left(w, \bar{w}, v_{i n} \mid Y-Y_{M}=\ln \left(M^{2} / s_{0}\right) \equiv Y_{m}\right)= \\
& \frac{w e^{-\Delta_{\mathbb{P}} Y}}{1+w\left(e^{-\Delta_{\mathbb{P}} Y_{m}}-1\right)}+\frac{\bar{w} e_{m}^{-\Delta_{\mathbb{P}} Y}}{1+\bar{w}\left(e^{-\Delta_{\mathbb{P}} Y_{m}}-1\right)}-\frac{\left(w+\bar{w}-v_{i n}\right) e^{-\Delta_{\mathbb{P}} Y_{m}}}{1+\left(w+\bar{w}-v_{i n}\right)\left(e^{-\Delta_{\mathbb{P}} Y_{m}}-1\right)} .
\end{array}
$$

To find the cross section for single diffractive production, we need to calculate the term which is proportional to $v_{i n}$, and to replace $v_{i n}$ by $2 \Delta_{\mathbb{P}} w \bar{w}$. Indeed, this term means that at $Y=Y_{m}$ we have only one cut Pomeron, while all other cut Pomerons at this rapidity have decayed to Pomerons without cuts. For simplicity we choose $Y^{\prime}=Y-Y_{M}=Y_{m}$ (see Fig. 6). Replacing $v_{i n} \rightarrow 2 \Delta_{\mathbb{P}} w \bar{w}$, means that at $Y=Y_{M}$, the last cut Pomeron splits into two Pomerons (see Fig. 6). Therefore,

$$
N_{s d}^{M F A}\left(w, \bar{w} ; Y-Y_{M}=\ln \left(M^{2} / s_{0}\right) \equiv Y_{m}\right)=2 \Delta_{\mathbb{P}} w \bar{w} \frac{e^{\Delta_{\mathbb{P}} Y_{m}}}{\left(1+(w+\bar{w})\left(e^{\Delta_{\mathbb{P}} Y_{m}}-1\right)^{2}\right)^{2}}
$$

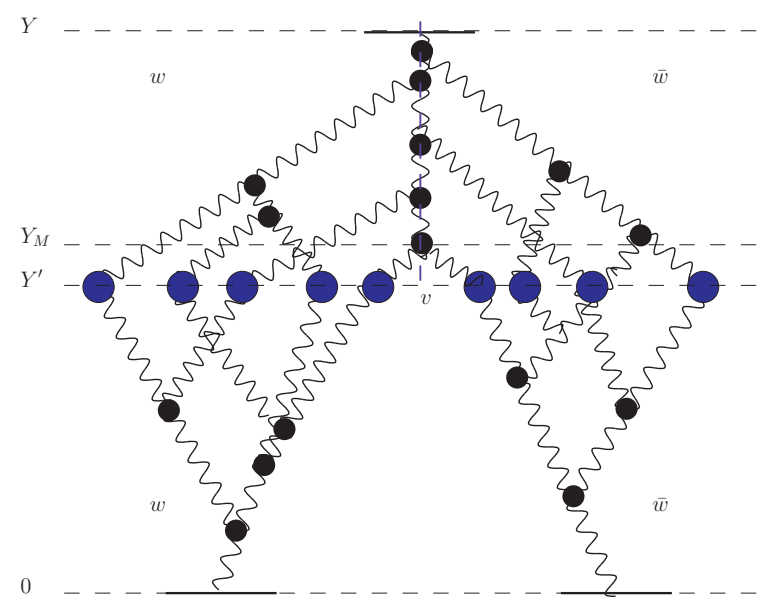

Figure 6: The MPSI approximation for the cross section of single diffractive production of mass $\left(M^{2}, Y-Y_{M}=\right.$ $\left.\ln \left(M^{2} / s_{0}\right)\right)$. The dashed lines shows the cut Pomerons. All other notations, are as in Fig. 团. 
The general Eq. (3.23) can be rewritten in this case in the form

$$
\begin{aligned}
& N_{s d}^{M P S I}\left(Y, Y_{m}=\ln \left(M^{2} / s_{0}\right)\right)= \\
& \left.\sum_{n=1 ; m=1}^{\infty} \frac{(-1)^{n+m}}{n ! m !} \gamma^{n+m} \frac{\partial^{n}}{\partial^{n} w^{p}} \frac{\partial^{m}}{\partial^{n} \bar{w}^{p}} N_{s d}^{M F A}\left(w^{p}, \bar{w}^{p} ; Y-Y_{M}=\ln \left(M^{2} / s_{0}\right) \equiv Y_{m}\right)\right|_{w=1 ; \bar{w}=1} \\
& \times\left.\left.\frac{\partial^{n} N^{M F A}\left(w^{t}, Y-Y_{m}\right)}{\partial^{n} w^{t}}\right|_{w^{t}=1} \frac{\partial^{m} N^{M F A}\left(\bar{w}^{t}, Y-Y_{m}\right)}{\partial^{n} \bar{w}^{t}}\right|_{\bar{w}=1} \\
& =1-\left\{\exp \left(-\gamma\left(\frac{\partial}{\partial w^{p}} \frac{\partial}{\partial w^{t}}+\frac{\partial}{\partial \bar{w}^{p}} \frac{\partial}{\partial \bar{w}^{t}}\right)\right) N_{s d}^{M F A}\left(w, \bar{w} ; Y_{m}\right)\right. \\
& \left.\times N^{M F A}\left(\bar{w}^{t}, Y-Y_{m}=Y_{M}\right) N^{M F A}\left(\bar{w}^{t}, Y-Y_{m}=Y_{M}\right)\right\}\left.\right|_{w^{p}=w^{t}=\bar{w}^{p}=\bar{w}^{t}=1} \\
& =\frac{\Delta_{\mathbb{P}} \gamma^{2}}{6} \frac{e^{\Delta_{P}\left(2 Y-Y_{m}\right)}}{L^{2}\left(Y, Y_{m}\right)} G\left(L\left(Y, Y_{m}\right)\right),
\end{aligned}
$$

where

$$
G(L)=L\left((L-1)^{2}-2\right)+e^{1 / L}(1+3 L) \Gamma_{0}(1 / L)
$$

and

$$
L\left(Y, Y_{m}\right)=\gamma \exp \left(\Delta_{\mathbb{P}}\left(Y-Y_{m}\right)\right)\left\{\exp \left(\Delta_{\mathbb{P}} Y_{m}\right)-1\right\} .
$$

$N_{s d}^{M P S I}\left(Y, Y_{m}=\ln \left(M^{2} / s_{0}\right)\right)$ describes the differential single diffraction cross section for the production of mass $M$. We can calculate the integrated diffraction cross section

$$
\begin{aligned}
N_{d i f f}^{M P S I}\left(Y ; M_{\max }, M_{\text {min }}\right) & \equiv \int_{y_{m}(\min )}^{y_{m}(\max )} d y_{m} N_{s d}^{M P S I}\left(Y, Y_{m}=\ln \left(M^{2} / s_{0}\right)\right) \\
& =\frac{\gamma}{6} e^{\Delta_{\mathbb{P}} Y}\left(B\left(L\left(Y, Y_{m}(\max )\right)\right)-B\left(L\left(Y, Y_{m}(\min )\right)\right)\right),
\end{aligned}
$$

where $Y_{m}(\max )=\ln \left(M_{\max }^{2} / s_{0}\right), Y_{m}(\min )=\ln \left(M_{\min }^{2} / s_{0}\right)$ and

$$
B(L)=2+\frac{1}{L^{2}}-\frac{1}{L}-\frac{e^{\frac{1}{L}} \Gamma_{0}(1 / L)}{L^{3}} .
$$

$M_{\max }$ and $M_{\min }$ denote the largest and the smallest masses which are produced in the diffractive process.

The expression for the integrated cross section for double diffraction can be obtained directly from the unitarity constraint of Eq. (2.4), as the diagrams that describe the elastic and single diffraction cross sections, do not contribute to the set of Pomeron diagrams, that describe the exact Pomeron Green's function (see Fig. 0 which contains examples of the diagrams that contribute to double diffractive production).

The unitarity constraint is given by

$$
2 N^{M P S I}=N_{d d}^{M P S I}+N_{i n}^{M P S I},
$$

where $N_{i n}^{M P S I}$ stands for the inelastic cross section. It was shown that $N_{i n}^{M P S I}$ is equal to $N^{M P S I}(2 T(y))$ (see Refs. [29,38,39]). Therefore, the integrated double diffraction cross section can be written in the form

$$
N_{d d}^{M P S I}(Y)=2 N^{M P S I}(T(Y))-N^{M P S I}(2 T(Y)) .
$$



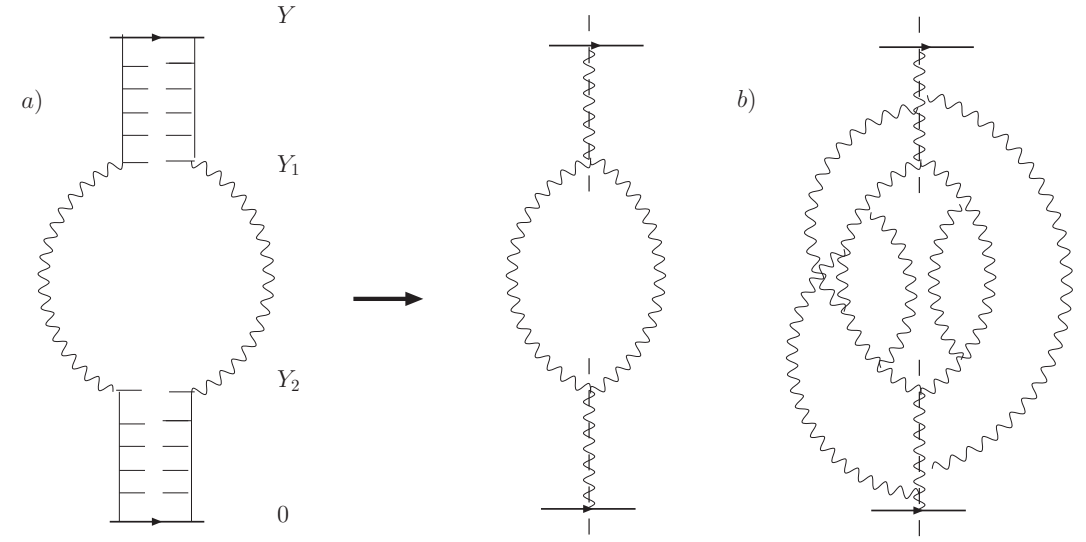

Figure 7: Several examples of the Pomeron diagrams that lead to the double diffractive production. Fig. 7 a is the simplest diagram that describes the process of double diffraction in the regions of large mass $Y-Y_{1}=\ln \left(M_{1}^{2} / s_{0}\right)$ and $Y_{2}=\ln \left(M_{2}^{2} / s_{0}\right)$. Fig. 5-b contains examples of more complicated diagrams in the region of large masses. The dashed line indicates the cut Pomeron which describes the production of hadrons (see Fig. ㄱa).

\subsection{Our approach}

In our approach we combine the G-W mechanism with the exact Pomeron Green's function of Eq. (3.30). First, we replace the bare Pomeron Green's function $G\left(Y-Y^{\prime}\right)=\exp \left(-\Delta_{\mathbb{P}}\left(Y-Y^{\prime}\right)\right)$ in Eq. (2.7) by $G_{\mathbb{P}}^{M P S I}\left(Y-Y^{\prime}\right)$ of Eq. (3.30), and, obtain

$$
\Omega_{i, k}(s, b)=g_{i} g_{k} G_{\mathbb{P}}^{M P S I}\left(Y-Y^{\prime}\right) S\left(b ; m_{i}, m_{k} ; \alpha_{\mathbb{P}}^{\prime} \ln \left(s / s_{0}\right)=0\right),
$$

with a profile function $S\left(b ; m_{i}, m_{k} ; \alpha_{I P}^{\prime} \ln \left(s / s_{0}\right)\right)$ determined by Eq. 2.10).

Using Eq. (2.12), Eq. (2.15) and Eq. (2.16) with $\Omega_{i, k}(s, b)$ from Eq. (3.42), we can calculate elastic and total cross sections. These formulae provide a correct description of low mass diffractive production due to the G-W mechanism. However, to include the Pomeron interactions in processes of diffractive production, we need to change Eq. (2.13) and Eq. (2.14). We first introduce (see Fig. 5-a)

$$
\Omega_{i, k}^{s d}(s, M ; b)=g_{i} g_{k}^{2} N_{s d}^{M P S}\left(Y=\ln \left(s / s_{0}\right), Y_{m}=\ln \left(M^{2} / s_{0}\right)\right) S^{s d}\left(b ; m_{i}, m_{k}\right),
$$

where the new profile function $S^{s d}\left(b ; m_{i}, m_{k}\right)$ is the Fourier transform of

$$
\frac{1}{\left.1+q^{2} / m_{i}^{2}\right)^{2}} \int \frac{d^{2} k}{(2 \pi)^{2}} \frac{1}{\left.1+(\vec{q}+\vec{k})^{2} / m_{i}^{2}\right)^{2}} \frac{1}{\left.1+(\vec{q}-\vec{k})^{2} / m_{i}^{2}\right)^{2}} \Longrightarrow S^{s d}\left(b ; m_{i}, m_{k}\right) .
$$

We found to within an accuracy of around $5 \%$, that the profile function $S^{s d}\left(b ; m_{i}, m_{k}\right)$ can be approximated by

$$
S^{s d}\left(b ; m_{i}, m_{k}\right)=\frac{m_{k}^{2}}{12 \pi} S\left(b ; m_{i}, \bar{m}_{k} ; \alpha_{\mathbb{P}}^{\prime} \ln \left(s / s_{0}\right)=0\right)
$$

where $\bar{m}_{k}^{2}=2 \sqrt{3} m_{k}^{2}$.

For the calculation of the integrated cross section of single diffractive production we define

$$
\begin{aligned}
& \Omega_{i, k}^{s d}\left(s, M_{\max }, M_{\min } ; b\right)= \\
& g_{i} g_{k}^{2} N_{\text {diff }}^{M P S}\left(Y=\ln \left(s / s_{0}\right), Y_{m}(\max )=\ln \left(M_{\max }^{2} / s_{0}\right), Y_{m}(\min )=\ln \left(M_{\min }^{2} / s_{0}\right)\right) S^{s d}\left(b ; m_{i}, m_{k}\right) .
\end{aligned}
$$


For the integrated cross section of the single diffraction channel, we obtain the following expression which takes into account both the G-W mechanism, and the enhanced Pomeron diagrams:

$$
\begin{aligned}
\sigma_{d i f f}=\int_{M_{\min }^{2}}^{M_{\max }^{2}} \frac{d M^{2}}{M^{2}} \frac{d \sigma^{s d}(s, M)}{d M^{2}} & =\int d^{2} b\left\{\alpha^{2} \beta^{2}\left(\alpha^{2} e^{-\frac{\Omega_{1,1}}{2}}-\left(\alpha^{2}-\beta^{2}\right) e^{-\frac{\Omega_{1,2}}{2}}-\beta^{2} e^{-\frac{\Omega_{2,2}}{2}}\right)^{2}\right. \\
& \left.+\left\{\alpha^{2} \Omega_{1,1}^{\text {diff }} e^{-\Omega_{1,1}}+\beta^{2} \Omega_{2,2}^{d i f f} e^{-\Omega_{2,2}}+2 \alpha^{2} \beta^{2} \Omega_{1,2}^{\text {diff }} e^{-\Omega_{1,2}}\right\}\right\},(3.4
\end{aligned}
$$

where $\Omega_{i, k}^{\text {diff }}$ are given by Eq. (3.46). The differential cross section for single diffraction in the region $M>M_{\min }$ is

$$
M^{2} \frac{d \sigma^{s d}(s, M)}{d M^{2}}=\int d^{2} b\left\{\alpha^{2} \Omega_{1,1}^{s d} e^{-\Omega_{1,1}}+\beta^{2} \Omega_{2,2}^{s d} e^{-\Omega_{2,2}}+2 \alpha^{2} \beta^{2} \Omega_{1,2}^{s d} e^{-\Omega_{1,2}}\right\},
$$

where $\Omega_{i, k}^{s d}$ are given by Eq. (3.43). In Eq. (3.47) and Eq. (3.49) we use $\Omega_{i, k}$ of Eq. (3.42).

For the double diffractive cross section we introduce

$$
\Omega_{i, k}^{d d}(s, b)=g_{i} g_{k} N_{d d}^{M P S I}(Y) S\left(b ; m_{i}, m_{k} ; \alpha_{I P}^{\prime} \ln \left(s / s_{0}\right)=0\right) .
$$

Using this $\Omega_{i, k}^{d d}$ we obtain for the integrated DD cross section

$$
\begin{aligned}
\sigma_{d d} & =\int_{0}^{\infty} \frac{d M_{1}^{2}}{M_{1}^{2}} \int_{0}^{\infty} \frac{d M_{2}^{2}}{M_{2}^{2}} \frac{d \sigma^{s d}\left(s, M_{1}, M_{2}\right)}{d M_{1}^{2} d M_{2}^{2}}=\int d^{2} b\left\{\alpha^{4} \beta^{4}\left(e^{-\frac{\Omega_{1,1}}{2}}-2 e^{-\frac{\Omega_{1,2}}{2}}+e^{-\frac{\Omega_{2,2}}{2}}\right)^{2}\right. \\
& \left.+\left\{\alpha^{4} \Omega_{1,1}^{d d} e^{-\Omega_{1,1}}+\beta^{4} \Omega_{2,2}^{d d} e^{-\Omega_{2,2}}+2 \alpha^{2} \beta^{2} \Omega_{1,2}^{d d} e^{-\Omega_{1,2}}\right\}\right\}
\end{aligned}
$$

For the integrated single and double diffractive production, the expressions each contains two terms: the first is responsible for G-W mechanism for these processes, while the second originates from the large mass diffraction of the enhanced Pomeron diagrams.

Eq. (2.12), Eq. (2.15) and Eq. (2.16) with $\Omega_{i, k}(s, b)$ from Eq. (3.42) and Eq. (3.43) - Eq. (3.52) give the full list of formulae in our approach. We wish to emphasize that our approach is based on the pQCD input for calculating the enhanced Pomeron diagrams, and on the G-W mechanism for low mass diffractive production.

\section{Results of the fit}

\subsection{Cross sections and elastic slope}

We have adjusted the parameters of our model which are listed in Table 2, using the formulae of section 3.6. The fit is based on 55 experimental data points, which includes the $p-p$ and $\bar{p}-p$ total cross sections, integrated elastic cross sections, integrated single and double diffraction cross sections, and the forward slope of the elastic cross section in the ISR-Tevatron energy range. The model gives a good reproduction of the data, with a $\chi^{2} /$ d.o.f. $\approx 1.25$. The quality of description of the experimental data is shown in 
Fig. 1, Fig. 2, Fig. 8, Fig. 9 and Fig. 10. A significant contribution to $\chi^{2} /$ d.o.f. stems from the uncertainty for the value of two single diffraction cross sections, and of the total cross section at the Tevatron. The $\chi^{2} /$ d.o.f. in Table 2 is calculated neglecting the contribution of the CDF measurement [41] ( $\sigma_{\text {tot }}=80 \mathrm{mb}$. at the Tevatron energy). The important advantage of our approach, is that the model provides a very good reproduction of the DD data points. In our previous attempt to describe the DD data [6] within a G-W approach, it was necessary to assume a non-factorizable contribution for the Pomeron exchange, resulting in marginally acceptable results.

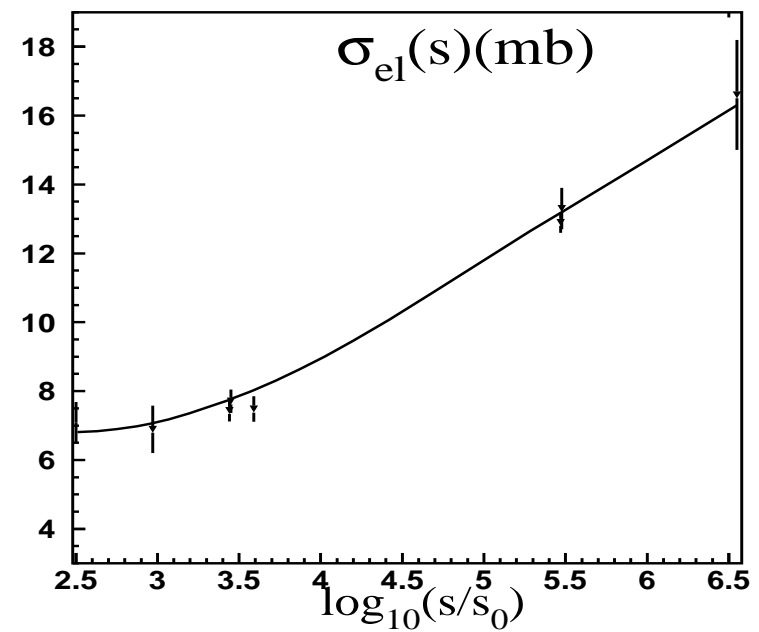

Figure 8: Energy dependence of $\sigma_{e l}$.

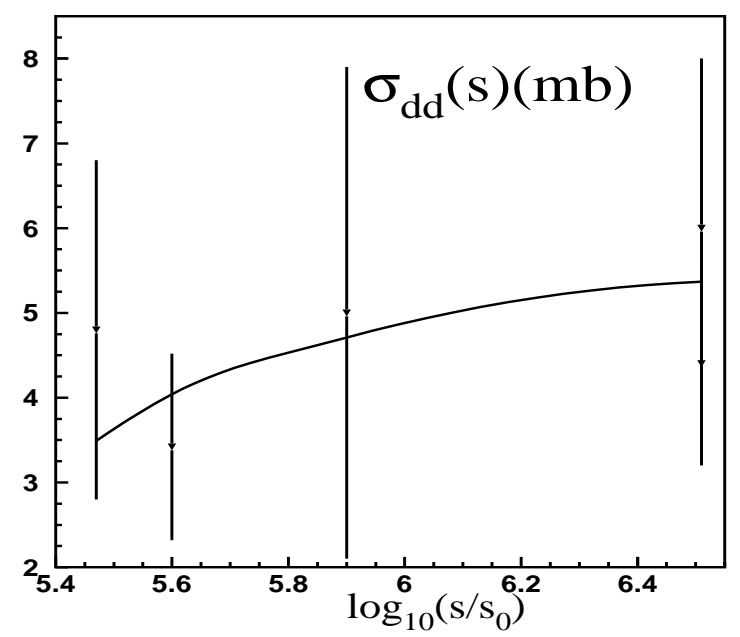

Figure 10: Energy dependence of $\sigma_{d d}$.

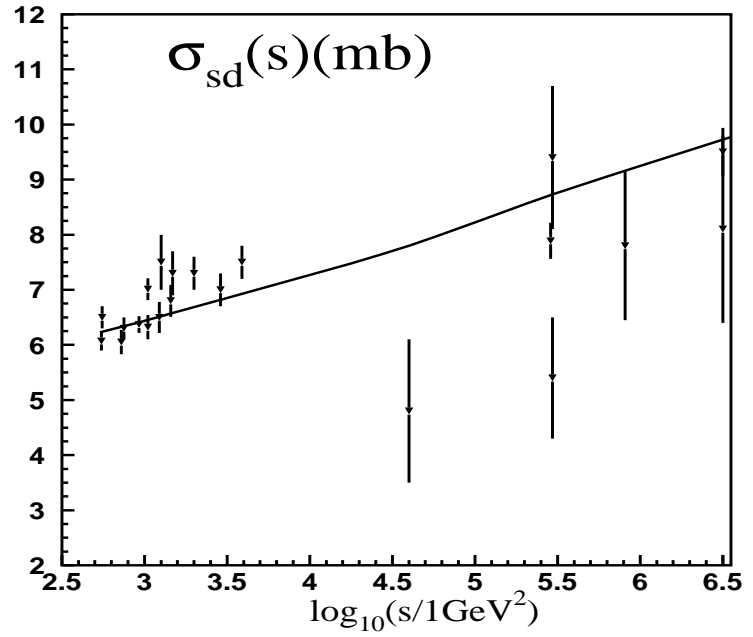

Figure 9: Energy dependence of the cross section $\sigma_{s d}$.

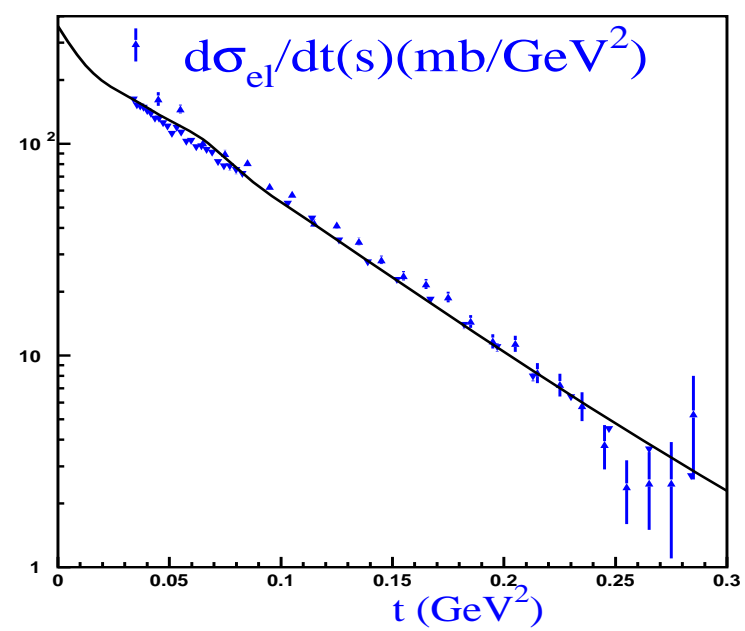

Figure 11: $t$ dependence of $d \sigma_{e l} / d t$ at the Tevatron $(W=1800 \mathrm{GeV})$. 


\begin{tabular}{|l|l|l|l|l|l|l|}
\hline$\Delta_{\mathbb{P}}$ & $\beta$ & $\alpha_{\mathbb{P}}^{\prime}$ & $g_{1}$ & $g_{2}$ & $m_{1}$ & $m_{2}$ \\
\hline 0.335 & 0.339 & $0.012 \mathrm{GeV}^{-2}$ & $5.82(0.90) \mathrm{GeV}^{-1}$ & $239.6(37.27) \mathrm{GeV}^{-1}$ & $1.54 \mathrm{GeV}$ & $3.06 \mathrm{GeV}$ \\
\hline$\Delta_{\mathbb{R}}$ & $\gamma$ & $\alpha_{\mathbb{R}}^{\prime}$ & $g_{1}^{\mathbb{R}}$ & $g_{2}^{\mathbb{R}}$ & $R_{0,1}^{2}$ & $\chi^{2} /$ d.o.f. \\
\hline-0.60 & 0.0242 & $0.6 \mathrm{GeV}^{-2}$ & $13.22 \mathrm{GeV}^{-1}$ & $367.8 \mathrm{GeV}^{-1}$ & $4.0 \mathrm{GeV}^{-2}$ & 1.0 \\
\hline
\end{tabular}

Table 2: Fitted parameters for our model which includes G-W mechanism for diffractive production, as well as the diffractive processes that stem from enhanced Pomeron diagrams (Pomeron loops). In our approach we have extracted a factor of $\gamma$ from the product $g_{i} g_{k}$. Therefore, the value of the Pomeron-hadron vertex is equal to $g_{i}=g_{i}($ from the table $) \times \sqrt{\gamma}$, and this value is shown in parentheses.

\section{$4.2 t$ - dependence of the differential elastic cross section}

The behaviour of the elastic cross section in the region of small $t$, is characterized by $B_{e l}$ and $\sigma_{t o t}$, which are included in the set of experimental data that we use for our fit. However, we wish to know the scattering amplitude at a relatively high value of $t \geq 0.1 \mathrm{GeV}^{2}$, where the simple exponential $t$ behaviour of the input elastic amplitude does not describe the data [6]. In Fig. 11 we plot our prediction with the parameters of Table 2 for the $t$-behaviour of the elastic cross section at the Tevatron energy $W=1800 \mathrm{GeV}$. We reproduce the data quite well, and it is a considerable improvement over the results obtained in [6]. $\ddagger$

\subsection{Mass dependence of the diffractive cross section}

In Fig. 12, we plot the cross section of single diffraction as a function of $1-x_{l}=M^{2} / s$ at the Tevatron energy. For this cross section, in the region of high mass, we use Eq. (3.49), while for diffraction in the region of low mass, we need to make some assumptions regarding the dependence of this cross section on $M$. Following Refs. [7,42] we assume that the main contribution for the G-W part of single diffractive production stems, from the $\mathbb{R} \mathbb{P} \mathbb{R}$ term, which does not depend on $x_{L}$. Therefore, the resulting contribution has the following form:

$$
\begin{aligned}
& M^{2} \frac{d \sigma_{s d}(s, M)}{d M^{2} d t}= \\
& =M^{2} \frac{d \sigma_{s d}^{\text {Low }} \mathrm{M}_{(s, M)}}{d M^{2} d t}(\mathbb{R I P} \mathbb{R} \text { term ; G-W contribution })+M^{2} \frac{d \sigma_{s d}^{\operatorname{High~} \mathrm{M}}(s, M)}{d M^{2} d t}(E q . \text { (3.49) })
\end{aligned}
$$

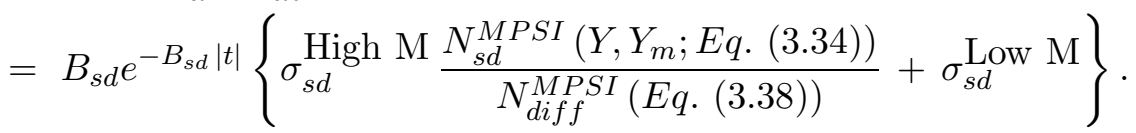

In our parametrization, the scale of the second term is determined by the G-W mechanism. The fact that the $\mathbb{R} \mathbb{P} \mathbb{R}$ term is responsible for $x_{L}$ behaviour, is an additional independent input. However, an argument for the $\mathbb{R} \mathbb{P} \mathbb{R}$ term, is that if $\Delta_{\mathbb{P}}=0$, then both $\mathbb{P} \mathbb{P} \mathbb{P}$ and $\mathbb{R} \mathbb{P} \mathbb{R}$ lead to a diffractive cross section, which is constant as a function of energy.

\footnotetext{
${ }^{\ddagger}$ We thank M. Ryskin who pointed out that the model of Ref. [6], gives a minimum at $|t| \approx 0.1 G e V^{2}$ which contradicts the experimental data.
} 


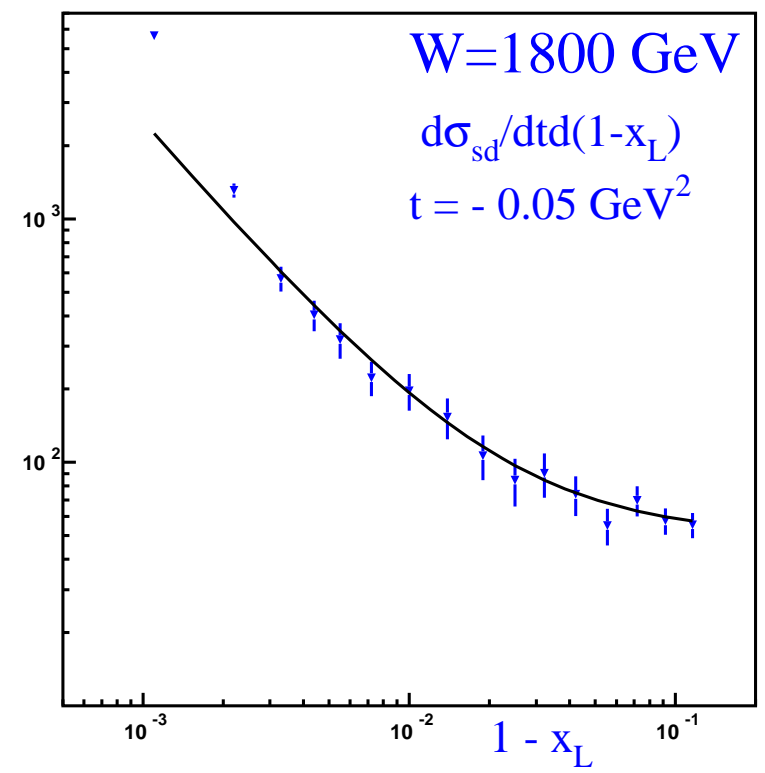

Figure 12: Dependence of single inclusive cross section on $1-x_{L}=M^{2} / s$, where $M$ is the mass of the diffractively produced system. Data are taken from Refs. [41, 42].

\section{Predictions for LHC and Cosmic Rays Energies: $b$-dependence of the amplitudes}

Fig. 13 shows our prediction for high energy behaviour of the total, elastic and diffractive cross sections as well as of the elastic slope.

At $\mathrm{W}=14 \mathrm{TeV}$ (LHC energy): $\sigma_{t o t}=92.1 \mathrm{mb}, \sigma_{e l}=20.9 \mathrm{mb}, \sigma_{s d}=11.8 \mathrm{mb}, \sigma_{d d}=6.08 \mathrm{mb}$ and $B_{e l}=20.6 \mathrm{GeV}^{-2}$. Comparing these results with the prediction of our previous model $\left(\sigma_{\text {tot }}=110.5 \mathrm{mb}\right.$, $\sigma_{e l}=25.3 \mathrm{mb}, \sigma_{s d}=11.6 \mathrm{mb}, \sigma_{d d}=4.9 \mathrm{mb}$ and $B_{e l}=20.5 \mathrm{GeV}^{-2}$ at the LHC energy) we see that the above comparison

leads to a few general observations, on which we will elaborate in the Discussion section.

1) The predictions of our Ref. [6] for $\sigma_{t o t}$ and $\sigma_{e l}$ at the LHC are considerably higher than the corresponding predictions obtained in the present analysis. The difference between the two sets of predictions grow monotonically with energy. Note, that the moderate growth of $\sigma_{t o t}$ and $\sigma_{e l}$ obtained in the present study continues up to energies as high as $10^{5} \mathrm{GeV}$, and probably higher. See Fig.13 and Table 3. We attribute this behaviour to the fact, that in our fit, $\alpha_{\mathbb{P}}^{\prime}$ is small but not zero.

2) These features reflect the fact that whereas our previous model has an output compatible with $\Delta_{\text {eff }} \approx$ 0.08 up to exceedingly high energies, the present model (in which Pomeron enhanced diagrams play a significant role), has an output $\Delta_{\text {eff }}$ which decreases monotonically with energy.

3) The qualitative features of $\sigma_{t o t}$ and $\sigma_{e l}$ in our two models are also seen in $\sigma_{s d}$. However, $\sigma_{d d}$ behaves differently, slowly approaching a constant value above LHC energies.

4) Our $B_{s d}$ slope is approximately two times less $B_{e l}$. At high energy we have only the results of fit to the diffraction experimental data given in Ref. [41]. The values of $B_{s d}$ from this fit are $B_{s d}=7.7 \pm 0.6(4.2 \pm$ 


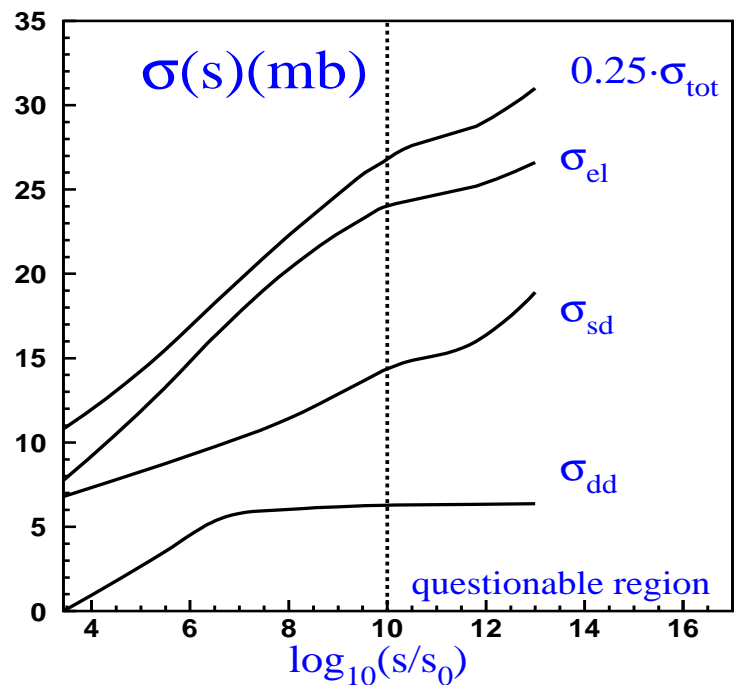

Fig. 13-a

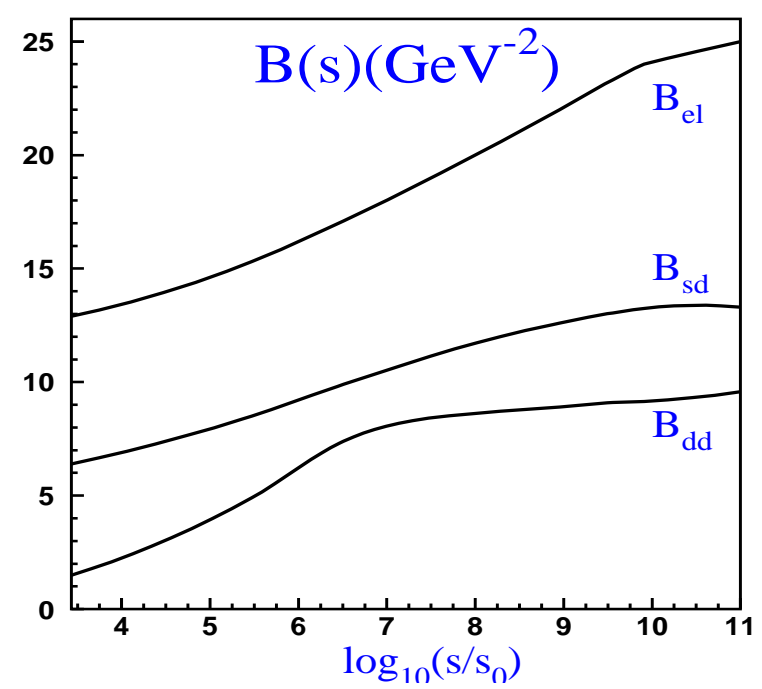

Fig. 13rb

Figure 13: The energy dependence of cross sections (Fig. 13 -a) and elastic slope $B_{e l}$ (Fig. 13-b) for elastic scattering and diffractive production. We have plotted $0.25 \sigma_{t o t}$, so as to show all the predictions on the same figure.

$0.5) \mathrm{GeV}^{-2}$ for $\mathrm{W}=546(1800) \mathrm{GeV}$. Our values (see Fig. 13-b) are $B_{s d}=8.5(9.9) \mathrm{GeV}^{-2}$ at these energies. The large difference in the experimental slopes have been discussed in Ref. [41].

To investigate the quantitative features of our present model further, we plot in Fig.14 the $b$ dependence of $A_{i, k}(s, b)$ and $a_{e l}(s, b)$ output amplitudes, at Tevatron and LHC energies. As seen, $A_{2,2}$ and $A_{1,2}$ reach the black bound at relatively low energies, whereas $A_{1,1}$ is below the black bound, approaching it very slowly.

As noted in Ref. [6], the output cross sections reach the unitarity bound when, and only when, $A_{1,1}=$ $A_{1,2}=A_{2,2}=1$. Since, $A_{1,1}$ grows very slowly with energy, we conclude that the very slow approach to the unitarity bound observed in Ref. [6], also occurs in the present model. Despite the fact that the two models have very different $t$ dependences, we hope that such structure of the amplitude does not depend on the model assumptions, but reflects the principle features of the hadron scattering at high energy. We shall expand on this issue in the Discussion section.

\section{Survival probability of diffractive Higgs production}

In the following we limit our discussion to the survival probability of Higgs production, in an exclusive central diffractive process. Most estimates of the values of survival probability have been made in the framework of G-W mechanism, in two channel eikonal models. A general review of such survival probability calculations can be found in Ref. [11]. The general formulae for the calculation of the survival probability 


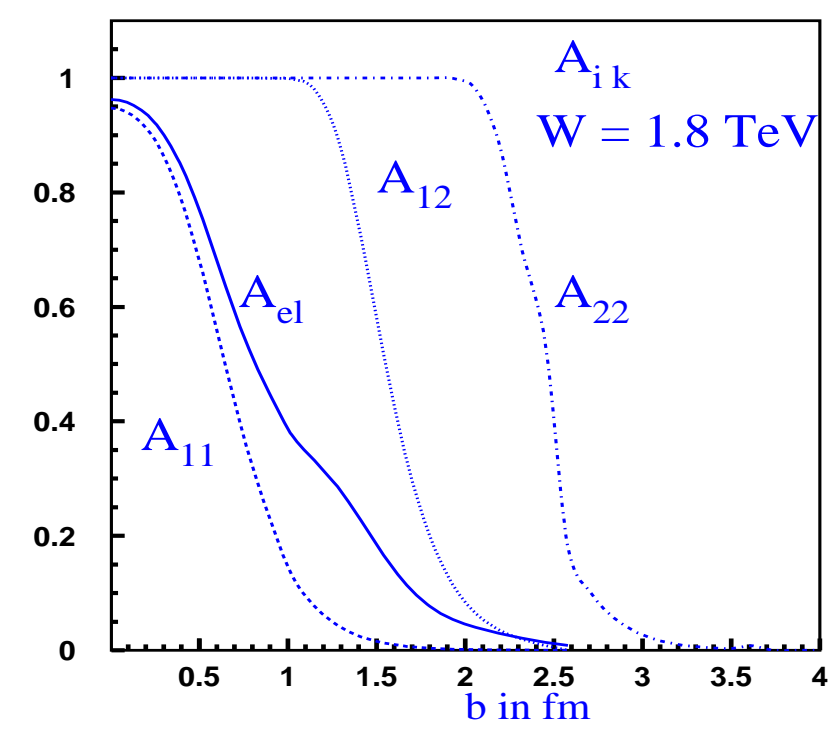

Fig. 14a

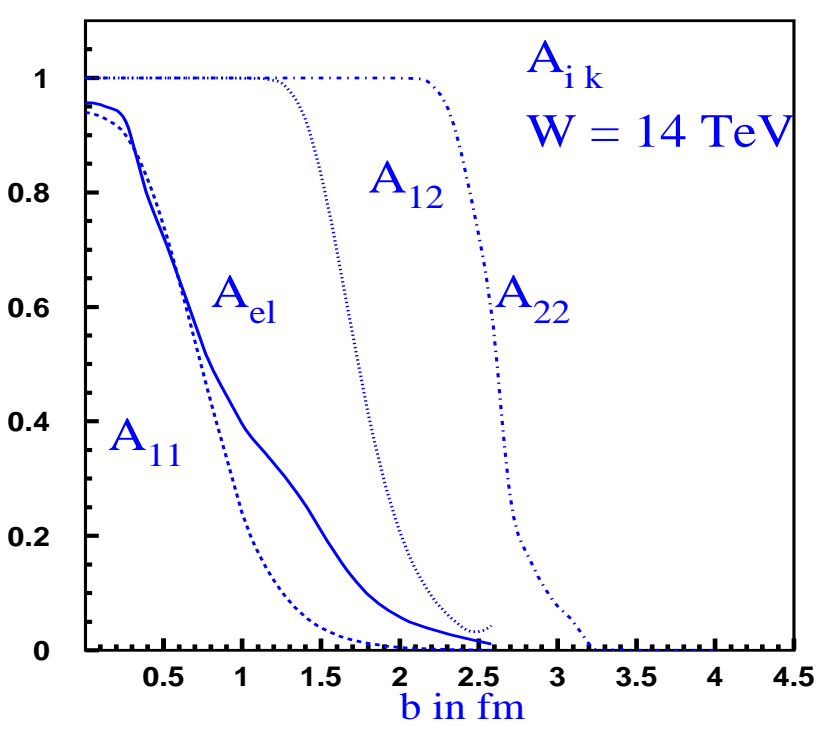

Fig. 14b

Figure 14: Impact parameter dependence of $A_{i, k}$ and $a_{e l}$ at different energies.

for diffractive Higgs boson production, have been discussed in Refs. [10-12]. The structure of the survival probability expression is shown in Fig. 15.-a. Accordingly,

$$
\left\langle\left|S_{2 c h}^{2}\right|\right\rangle=\frac{N(s)}{D(s)}
$$

where,

$$
\begin{gathered}
N(s)=\int d^{2} b_{1} d^{2} b_{2}\left[\sum_{i, k}<p\left|i>^{2}<p\right| k>^{2} A_{H}^{i}\left(s, b_{1}\right) A_{H}^{k}\left(s, b_{2}\right)\left(1-A_{S}^{i, k}\left(\left(s,\left(\mathbf{b}_{1}+\mathbf{b}_{2}\right)\right)\right)\right]^{2}\right. \\
D(s)=\int d^{2} b_{1} d^{2} b_{2}\left[\sum_{i, k}<p\left|i>^{2}<p\right| k>^{2} A_{H}^{i}\left(s, b_{1}\right) A_{H}^{k}\left(s, b_{2}\right)\right]^{2} .
\end{gathered}
$$

$<p \mid i>$ is equal to $\left\langle\Psi_{\text {proton }} \mid \Psi_{i}\right\rangle$ and, therefore, $\langle p| 1>=\alpha$ and $<p \mid 2>=\beta$.

$A_{s}$ denotes the soft strong interaction amplitude given by Eq. (2.7), Eq. (2.12), Eq. (2.13) and Eq. (2.14). The form of $A_{H}(s, b)$ has been discussed in Refs. [10,11]. In our model we assume an input Gaussian $b$ dependence for the hard amplitudes.

$$
A_{i, k}^{H}=A_{H}(s) \Gamma_{i, k}^{H}(b),
$$

where $A_{H}(s)$ is an $s$ - dependent arbitrary function which does not depend on $i, k$, and $\Gamma_{i, k}^{H}(b)=\frac{1}{\pi\left(R_{i, k}^{H}\right)^{2}} e^{-\frac{2 b^{2}}{\left(R_{i, k}^{H}\right)^{2}}}$. The hard vertices and radii $R_{i, k}^{H^{2}}$, are constants derived from HERA $J / \Psi$ elastic and inelastic photo and DIS production [40]. 


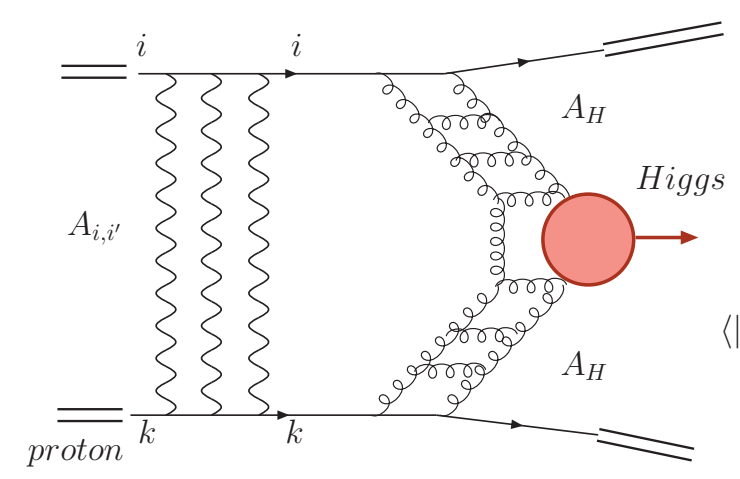

a)

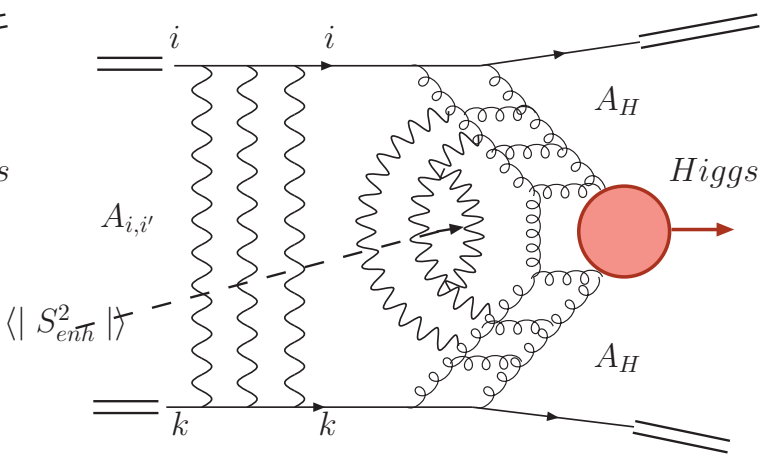

b)

Figure 15: Survival probability for exclusive central diffractive production of the Higgs boson. Fig. 15-a shows the contribution to the survival probability in the G-W mechanism, while Fig. 15-b illustrates the origin to the additional factor $\left\langle\left|S_{e n h}^{2}\right|\right\rangle$.

Following Refs. [11,12] we have introduced in the above, two hard $b$-profiles

$$
\begin{aligned}
& A_{H}^{p p}(b)=\frac{V_{p \rightarrow p}}{2 \pi B_{e l}^{H}} \exp \left(-\frac{b^{2}}{2 B_{e l}^{H}}\right), \\
& A_{H}^{p d}(b)=\frac{V_{p \rightarrow d}}{2 \pi B_{i n}^{H}} \exp \left(-\frac{b^{2}}{2 B_{i n}^{H}}\right) .
\end{aligned}
$$

The values $B_{e l}^{H}=3.6 \mathrm{GeV}^{-2}$ and $B_{i n}^{H}=1 \mathrm{GeV}^{-2}$, have been taken from the experimental ZEUS data on $J / \Psi$ production at HERA (see Refs. [11,43]).

Using Eq. (2.12)-Eq. (2.14), the integrands of Eq. (6.2) and Eq. (6.3) are reduced by eliminating common $s$-dependent expressions,

$$
\begin{gathered}
N(s)=\int d^{2} b_{1} d^{2} b_{2}\left[A_{H}\left(s, b_{1}\right) A_{H}\left(s, b_{2}\right)\left(1-A_{S}\left(\mathbf{b}=\mathbf{b}_{1}+\mathbf{b}_{2}\right)\right)\right]^{2} \\
=\int d^{2} b_{1} d^{2} b_{2}\left[\left(1-a_{e l}(s, b)\right) A_{H}^{p p}\left(b_{1}\right) A_{H}^{p p}\left(b_{2}\right)-a_{s d}(s, b)\left(A_{H}^{p d}\left(b_{1}\right) A_{H}^{p p}\left(b_{2}\right)\right.\right. \\
\left.\left.+A_{H}^{p p}\left(b_{1}\right) A_{H}^{p d}\left(b_{2}\right)\right)-a_{d d}(s, b) A_{H}^{p d}\left(b_{1}\right) A_{H}^{p d}\left(b_{2}\right)\right]^{2}, \\
D=\int d^{2} b_{1} d^{2} b_{2}\left[A_{H}^{p p}\left(b_{1}\right) A_{H}^{p p}\left(b_{2}\right)\right]^{2} .
\end{gathered}
$$

Eq. (6.1) does not give a correct estimate for the survival probability, and should be multiplied by a factor $\left(\left\langle\left|S_{e n h}^{2}\right|\right\rangle\right)$ that, incorporates the possibility for the Higgs boson to be emitted from the enhanced diagrams (see Fig. 15-b). Therefore, the resulting survival probability can be written as

$$
\left\langle\left|S^{2}\right|\right\rangle=\left\langle\left|S_{\text {enh }}^{2}\right|\right\rangle(\text { Eq. (6.15) }) \times\left\langle\left|S_{2 c h}^{2}\right|\right\rangle(\text { Eq. 6.1) }) .
$$


The first attempt to find $\left\langle\left|S_{\text {enh }}^{2}\right|\right\rangle$ was made in Refs. [34,44], where this factor was calculated neglecting the fact that the Higgs boson could be produced from the two gluon scattering with a difference in rapidity $\delta Y_{H}=\ln \left(M_{H}^{2} / s_{0}\right)$ (see for example Refs. [44,45]). The MPSI approach for this case is shown in Fig. 16, and it leads to the result:

$$
\left\langle\left|S_{e n h}^{2}\right|\right\rangle(Y)=\frac{\partial}{\partial T} N_{e l}^{M P S I}(T)=\frac{1}{T^{3}(Y)}\left\{-T(Y)+e^{\frac{1}{T(Y)}}(1+T(Y)) \Gamma_{0}\left(\frac{1}{T(Y)}\right)\right\}
$$

where $N_{e l}^{M P S I}(T)$ is given Eq. (3.28). It was originally suggested to divide this factor by $\left\langle\left|S_{e n h}^{2}\right|\right.$ \rangle$\left(Y=\delta Y_{H}\right)$

$$
\left\langle\left|S_{e n h}^{2}\right|\right\rangle(Y)=\frac{\left\langle\left|S_{e n h}^{2}\right|\right\rangle(Y ; E q .(6.10))}{\left\langle\left|S_{e n h}^{2}\right|\right\rangle\left(Y=\delta Y_{H} ; E q .(6.10)\right)} .
$$

In this paper we take into account $\delta Y_{H}$ in a consistent way, which was outlined in Ref. [34], and presented in Fig. 17 [16]. At rapidity $Y-Y^{\prime}-\frac{1}{2} \delta Y_{H}$, one of the partons (Pomerons) will produce a Higgs boson, and it should be removed from the cascade evolution. Therefore, those partons which will participate in the evolution will be characterized by a new generating function,

$$
\begin{aligned}
& \tilde{Z}\left(Y-Y^{\prime}-\frac{1}{2} \delta Y_{H}, u\right)= \\
& e^{-\Delta_{\mathbb{P}}\left(Y-y^{\prime}-\frac{1}{2} \delta Y_{H}\right)} \frac{\partial Z^{M F A}\left(Y-Y^{\prime}-\frac{1}{2} \delta Y_{H}, u\right)}{\partial u}=\frac{1}{\left(u+(1-u) e^{-\Delta_{\mathbb{P}}\left(Y-Y^{\prime}-\frac{1}{2} \delta Y_{H}\right)}\right)^{2}} .
\end{aligned}
$$

$\tilde{Z}\left(Y-Y^{\prime}-\frac{1}{2} \delta Y_{H} ; u\right)$ should be evolved to rapidity $Y-Y^{\prime}$ using Eq. (3.24). This evolution results in an improved generating function

$$
\widetilde{Z}\left(Y-Y^{\prime}, u\right)=\frac{\left(u+(1-u) e^{\frac{1}{2} \Delta_{\mathbb{P}} \delta Y_{H}}\right)^{2}}{\left(u+(1-u) e^{\Delta_{\mathbb{P}}(Y-Y)}\right)^{2}} .
$$

Note that for central Higgs boson production $\frac{1}{2} Y=Y^{\prime}$.

Symmetrically, using Eq. (6.12) we need to find $\tilde{Z}\left(Y^{\prime}-\frac{1}{2} \delta Y_{H}, u\right)$, and from Eq. (6.13), $\tilde{Z}\left(Y^{\prime}, u\right)$. The result for this function is

$$
\widetilde{\widetilde{Z}}\left(Y^{\prime}, u\right)=\frac{\left(u+(1-u) e^{\frac{1}{2} \Delta_{\mathbb{P}} \delta Y_{H}}\right)^{2}}{\left(u+(1-u) e^{\Delta_{\mathbb{P}} Y^{\prime}}\right)^{2}}
$$


Using these generating functions we obtain

$$
\begin{aligned}
& \left\langle\left|S_{\text {enh }}^{2}(M P S I)\right|\right\rangle(Y)= \\
& =\left.\left.\sum_{n=1}^{\infty} \frac{(-1)^{n+1}}{n !} \gamma^{n} \frac{\partial^{n} \widetilde{\widetilde{Z}}\left(Y-Y^{\prime}, u^{p}\right)}{\partial^{n} u^{p}}\right|_{u^{p}=1} \frac{\left.\partial^{n} \widetilde{\widetilde{Z}}\left(Y^{\prime}, u\right) u^{t}\right)}{\partial u_{t}}\right|_{u^{t}=1} \\
& =S(\mathcal{T}(Y))-2 e^{-\Delta_{\mathbb{P}}\left(Y-\delta Y_{H}\right) / 2} S 1(\mathcal{T}(Y))+e^{-2 \Delta_{\mathbb{P}}\left(Y-\delta Y_{H}\right) / 2} S 2(\mathcal{T}(Y) ; \\
& S(T)=\frac{1}{T^{3}}\left\{-T+e^{\frac{1}{T}}(1+T) e^{\frac{1}{T}} \Gamma_{0}\left(\frac{1}{T}\right)\right\} ; \\
& S 1(T)=\frac{1}{T^{3}}\left\{-T(1+T)+(1+2 T) e^{\frac{1}{T}} \Gamma_{0}\left(\frac{1}{T}\right)\right\} ; \\
& S 2(T)=\frac{1}{T^{3}}\left\{T\left[(T-1)^{2}-2\right]+(1+3 T) e^{\frac{1}{T}} \Gamma_{0}\left(\frac{1}{T}\right)\right\} .
\end{aligned}
$$

where

$$
\mathcal{T}(Y)=\gamma\left(e^{\Delta_{\mathbb{P}}\left(Y-Y^{\prime}\right)}-1\right)\left(e^{\Delta_{\mathbb{P}} Y^{\prime}}-1\right)
$$

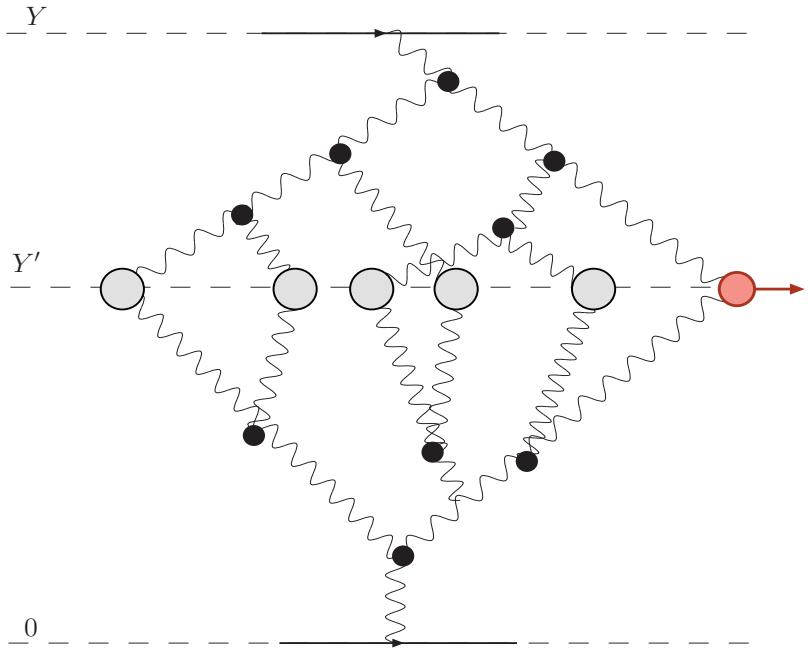

Figure 16: MPSI approach for $\left\langle\left|S_{e n h}^{2}\right|\right\rangle$ in the case when we neglect $\delta Y_{H}=\ln \left(M_{H}^{2} / s_{0}\right)$.

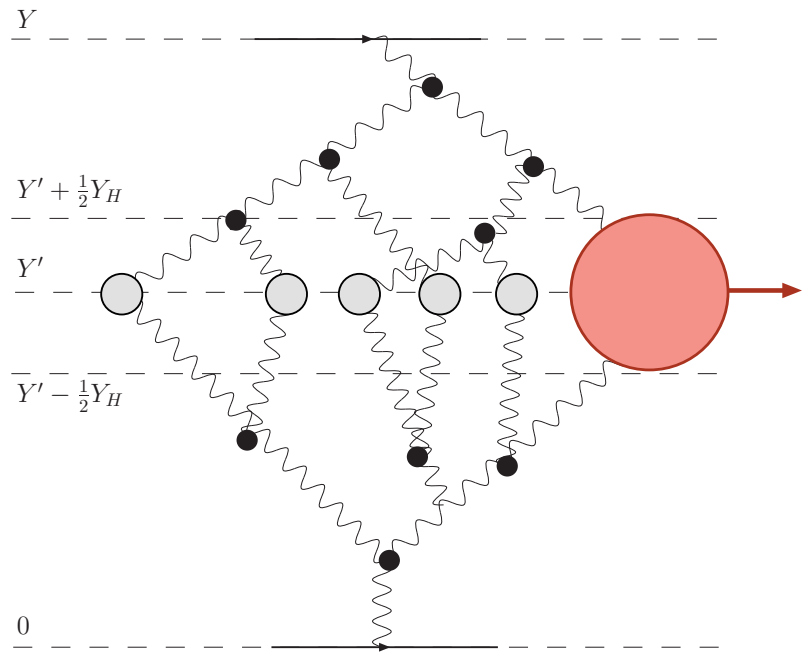

Figure 17: MPSI approach for $\left\langle\left|S_{e n h}^{2}\right|\right\rangle$ in the case when we take into account $\delta Y_{H}=\ln \left(M_{H}^{2} / s_{0}\right)$.

Using Eq. (6.9)-Eq. (6.18), we calculate the survival probability $\left\langle\left|S^{2}\right|\right\rangle$ for exclusive Higgs production in central diffraction. Our results are plotted in Fig. 18. In the following we focus on a detailed discussion of our LHC predictions based on the above. Calculating in the framework of a two amplitude eikonal model, $\left\langle\left|S_{2 c h}^{2}\right|\right\rangle$ for exclusive Higgs production in central diffraction is equal to $2.35 \%$, which is close to the value of this part of the survival probability estimated by the Durham group [5,7], and by our group [11]. However, the additional factor $\left\langle\left|S_{\text {enh }}^{2}\right|\right\rangle=0.063$ as derived from Eq. (6.15), leads to a final $\left\langle\left|S^{2}\right|\right\rangle=0.15 \%$. This result reflects a tendency for the value of the survival probability to be much smaller, than when evaluated in the two channel models $[6,44]$. Note, that the approximate expression for 


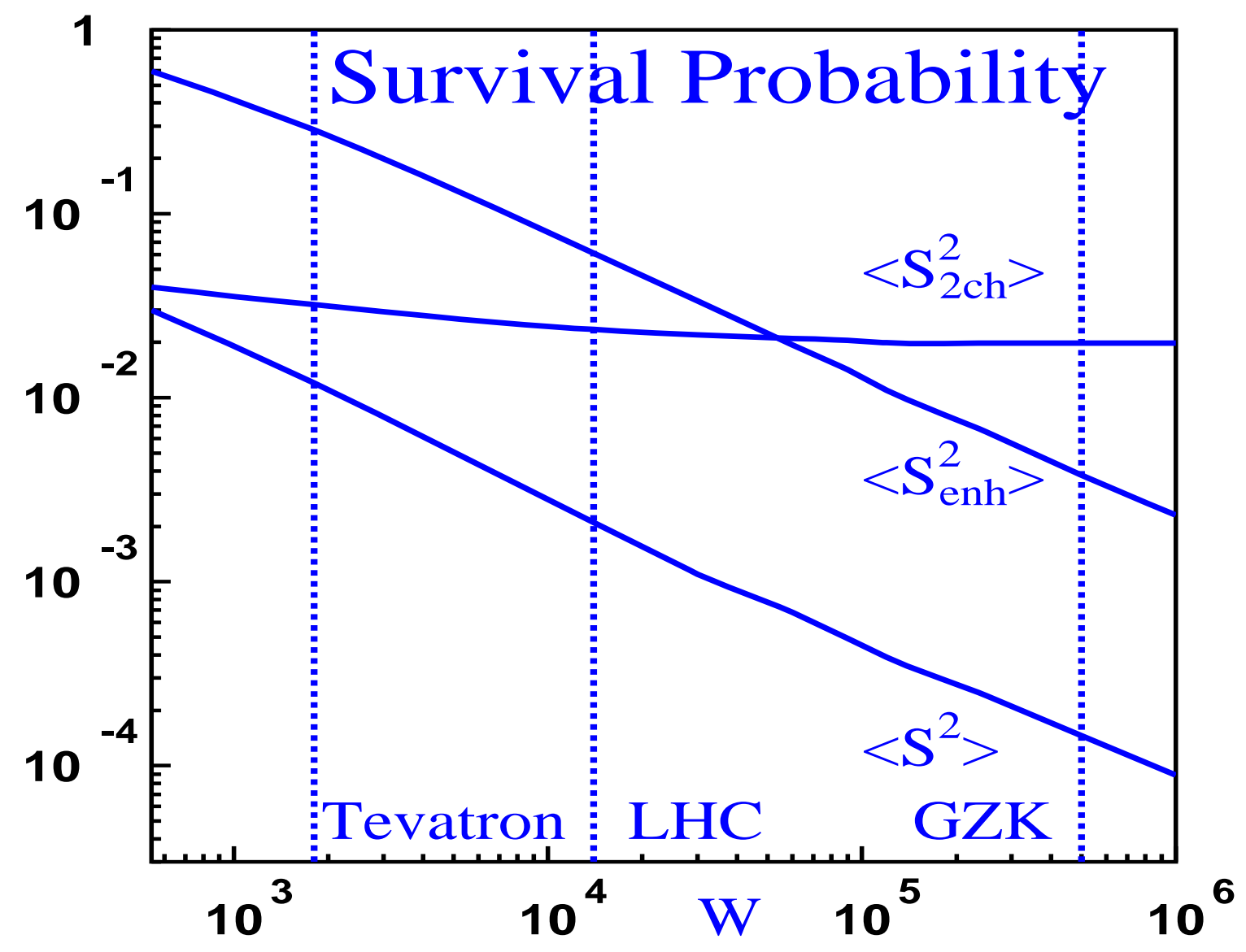

Figure 18: Energy dependence of centrally produced Higgs survival probability.

$\left\langle\left|S_{\text {enh }}^{2}\right|\right\rangle$ derived from Eq. (6.10) with our parameters, turns out to be three times smaller. In Ref. [7] the Pomeron diagrams were taken into account, but nevertheless, their contribution in the calculation of $\left\langle\left|S^{2}\right|\right\rangle$ was omitted, without any explanation.

\section{Discussion}

In this paper we have presented an approach for soft interactions at high energies based on two ingredients: 1) The Good-Walker mechanism for elastic and low mass diffraction.

2) Pomeron enchanced contributions which leads to the exact Pomeron Green's function, which is significantly different from one Pomeron exchange. This component provides the main contribution to high mass diffraction. 


\begin{tabular}{|c|c|c|c|c|c|c|}
\hline & \multicolumn{2}{|c|}{ Tevatron } & \multicolumn{2}{|c|}{$\overline{\mathrm{LHC}}$} & \multicolumn{2}{|c|}{$\bar{W}=10^{5} \mathrm{GeV}$} \\
\hline & GLM & KMR & GLM & KMR & GLM & KMR \\
\hline$\sigma_{\text {tot }}(\mathrm{mb})$ & 73.29 & 74.0 & 92.1 & 88.0 & 108.0 & 98.0 \\
\hline$\sigma_{e l}(\mathrm{mb})$ & 16.3 & 16.3 & 20.9 & 20.1 & 24. & 22.9 \\
\hline$\sigma_{s d}(\mathrm{mb})$ & 9.76 & 10.9 & 11.8 & 13.3 & 14.4 & 15.7 \\
\hline$\sigma_{s d}^{\text {low }} \mathrm{M}$ & 8.56 & 4.4 & 10.52 & 5.1 & 12.2 & 5.7 \\
\hline$\sigma_{s d}^{\text {high M }}$ & 1.2 & 6.5 & 1.28 & 8.2 & 2.2 & 10.0 \\
\hline$\sigma_{d d}(\mathrm{mb})$ & 5.36 & 7.2 & 6.08 & 13.4 & 6.29 & 17.3 \\
\hline$\left(\sigma_{e l}+\sigma_{s d}+\sigma_{d d}\right) / \sigma_{t o t}$ & 0.428 & 0.464 & 0.421 & 0.531 & 0.412 & 0.57 \\
\hline$S_{2 c h}^{2}(\%)$ & 3.2 & $2.7-4.8$ & 2.35 & $1.2-3.2$ & 2.0 & $0.9-2.5$ \\
\hline$S_{e n h}^{2}(\%)$ & 28.5 & 100 & 6.3 & 100 & 3.3 & 100 \\
\hline$S^{2}(\%)$ & 1.2 & $2.7-4.8$ & 0.21 & $1.2-3.2$ & 0.05 & $0.9-2.5$ \\
\hline
\end{tabular}

Table 3: Comparison of the GLM ( this paper) and KMR [7] models.

Our enhanced Pomeron formalism, is based on the observation that the soft scattering cross sections and slopes, can be reproduced with $\alpha_{\mathbb{P}}^{\prime} \approx 0.01 \mathrm{GeV}^{-2}$, rather than $\alpha_{P}^{\prime}=0.25 \mathrm{GeV}^{-2}$ typical of conventional Regge phenomenology. Our result of a very small $\alpha_{I P}^{\prime}$, implies that the observed shrinkage of the forward differential cross sections, traditionally associated with $\alpha_{\mathbb{P}}^{\prime}>0$, can be also reproduced with $\alpha_{\mathbb{P}}^{\prime} \approx 0$ coupled to strong screening, which produces the desired shrinkage.

In the following we discuss further the properties of our model, by comparing it with the KMR model [7], which is conceptually similar to ours, having the same two mechanisms: G-W and multiPomeron interactions. In the KMR model $\alpha_{I P}^{\prime} \equiv 0$ is an input assumption. In the present GLM model $\alpha_{\mathbb{P}}^{\prime}$ is a fitted parameter, and its value $\alpha_{\mathbb{P}}^{\prime}=0.012$ is an output. As we have discussed the small value of $\alpha_{I P}^{\prime}$, led us to our key hypothesis that we advocate in this paper: the soft processes are not so soft, but stem from short distances, where the QCD coupling is small ( $\alpha_{S} \approx 0.12$ to 0.16$)$. Using this hypothesis we built our theoretical approach. This approach is self consistent based on pQCD. This enables us to restrict our summation only to triple Pomeron vertices. KMR summation is based on an ad hoc assumption

$$
\Gamma(n \text { Pomerons } \rightarrow m \text { Pomerons }) \propto n m \lambda^{m+n-2} .
$$

Further, KMR claim that Eq. (7.1) leads to their main equation (Eq.(26) of Ref. [7]), which corresponds to the parton model. Both claims maybe correct, but they have not been proven in the KMR paper. A formal difficulty also noted by the KMR authors (at the beginning of their section 4.3) is that the formulae for diffractive production are ad hoc, and are not actually compatible with our Eq. (7.1) and Eq.(26) of Ref. [7].

In spite of the fact, that we do not think that KMR model is able to provide reliable estimates, it is interesting to compare our results, since both are based on the same physics.

1) The introduction of Pomeron induced interactions to the calculation, results in the accumulation of Pomeron loops along the initial Pomeron propogator, which lead to a monotonic reduction of the output 
$\Delta_{\text {eff }}$ with energy. In the KMR model, where additional diagrams, and not only diagrams with triple Pomeron interactions have been included, this process occurs more rapidly than in our model. Accordingly, they choose a very high input value, $\Delta_{i n}=0.55$. In our fit we have $\Delta_{i n}=0.335$. Both in the KMR model and in our model the effective shrinkage of the diffraction peak, stems from the Pomeron interactions. The difference is that $R^{2}(s)$ in the GLM model grows as $l n^{2} s$ with a coefficient proportional to $\alpha_{P}^{\prime}$. Since in evaluating our summations, we have made an approximation in which $\alpha_{I P}^{\prime}=0$, our calculations are only trustworthy up to $W \approx 10^{5} \mathrm{GeV}$. The high energy output of GLM and KMR models are presented in Table 3. For $\sigma_{d d}$ the contribution to the diffractive channels coming from the Pomeron enhanced diagrams, are larger in KMR than in GLM. Note that $\sigma_{d d}$, as calculated by GLM, saturates just above LHC energies, while in KMR it continues growing even at energies of $\mathrm{W}=10^{5} \mathrm{GeV}$, where it is predicted to be much larger than the $\sigma_{s d}$.

2) In Table 3 we define $\sigma_{s d}^{\text {Low } M}$ as the contribution of G-W mechanism, while in the KMR model low and high mass diffraction is allocated to mass values $M<M_{0}=2.5 \mathrm{GeV}$ and $M>M_{0}=2.5 \mathrm{GeV}$.

3) The behaviour of the ratio $R_{D}=\frac{\left(\sigma_{e l}+\sigma_{s d}+\sigma_{d d}\right)}{\sigma_{t o t}}$ conveys information regarding the onset of unitarity constraints at high enough energy. In the G-W model the Pumplin bound $R_{D} \leq 0.5$ is relevant. The multi-Pomeron induced contributions are not included in this bound. In the GLM model, $R_{D}<0.5$ and decreases very slowly with energy. It's corresponding $R_{D}^{G-W} \approx 0.35$, is a constant similar to the output of Ref. [6]. In KMR, $R_{D}>0.5$ and grows with energy. The difference between GLM and KMR appears to be due to the different summations of the Pomeron induced diagrams, incorporated in the models.

4) The difference in the values of the survival probabilities calculated in the two channel models are significant. The KMR estimate should be reduced by $S_{e n h}^{2}$ which is ignored in their calculation. Including this factor brings the GLM and KMR numbers closer.

5) The data analysis aimed at determining the three opacities $\Omega_{i, k}$, is conceptually different in the GLM and KMR models. In principle this information should be obtained utilizing Eq.(2.12)-Eq.(2.18). However, the experimental information we have at the UA(4)-Tevatron energies is not sufficient to constrain the Pomeron parameters. In the GLM model we, thus, included both the Pomeron and Reggeon trajectories, covering also the extensive ISR data. This enabled us to extract the Pomeron (fitted) parmeters. KMR have adopted a different strategy, obtaining the Pomeron opacities from a good fit to $\frac{d \sigma_{e l}}{d t}$ in UA(4)-Tevatron energy range. This implies a good reproduction of $\sigma_{t o t}, \sigma_{e l}$ and $B_{e l}$. From their paper [7], it is not clear whether KMR also fit the diffractive channels. This is not the only ambiguity in the KMR presentation of their results. Some of their parameters are explicitly presented and fitted, some are presented and "tuned", some are assumed and some are implied, but not explicitly presented.

6) As noted, we attribute our dynamical result to the output of our fit, in which $\alpha_{\mathbb{P}}^{\prime}$ is small but not zero! Both our model and KMR, predict total and elastic cross sections which are significantly smaller than in two channel eikonal estimates, which do not include the Pomeron enhanced contributions (see Refs. [4-6]). The differences are sufficiently large, so that measurements at LHC and Auger should be able to discrimenate between the various approaches. See details below.

7) The most practical, and perhaps the most interesting result we have obtained, is the small value (about $0.15 \%)$ for the survival probability of central diffractive Higgs production at the LHC. The very small value of the final $S_{H}^{2}$, is due to the smallnes of $S_{2 c h}^{2}$, multiplied by the small $S_{e n h}^{2}$. Our calculation does 
not include further reductions of $S_{H}^{2}$, due to additional short distance processes (see Refs. [44,46]). These may further reduce the result we obtained for the survival probability.

The region of applicability for our formulae is given by Eq. 3.19), in which, $Y \ll 1 / \gamma=41$, and by the fact that in our procedure for summing Pomeron diagrams we considered $\alpha_{\mathbb{P}}^{\prime}=0$. The first restriction leads to a very large kinematic range for rapidity. The seco

that $\alpha_{\mathbb{P}}^{\prime} \ln \left(s / s_{0}\right)<0.25 / m_{1}^{2}$ in our parameterization, leads to $Y<25$. This region, is marked as questionable in Fig. 13-a. The set of formulae in section 3.6 do not include the contribution of the 'fan' diagram to the elastic amplitude. In our context, it is important that the contribution of such diagrams which determines the high mass diffraction, turns out to be small. In the following we list a few experimental signatures, which should be measured at the LHC in the near future. These will give us a clue regarding the veracity of the models discussed in the paper:

i) Measurements of $\sigma_{t o t}$ and $\sigma_{e l}$ should serve as a critical test for the relevance of Pomeron enhanced diagrams. The difference in predictions for models including (excluding) Pomeron enhanced diagrams, becomes even more significant at Cosmic Ray energies. The Auger experiment, where we expect results for cross sections in the near future, at energies in the $10^{5} \mathrm{GeV}$ range, should allow us to discriminate between the alternative approaches.

ii) The Pomeron enhanced contribution to the diffractive channels, as calculated by KMR, is considerably larger than our predictions. This is significant for $\sigma_{d d}$, which acquires a large value in the KMR approach. iii) An early estimate of the value of $S_{H}^{2}$ should be obtained by an LHC measuremnt of the role of hard central LRG dijet production in a GJJG configuration, when compared to the pQCD prediction.

To summarize, we developed an approach which is self consistent, and is based on a perturbative QCD input. We hope that a more microscopic approach with roots in QCD saturation, can be built and we believe that this paper will contribute to such an effort.

\section{Acknowledgements}

We are grateful to Omry Netzer and Andrey Kormilitzin for useful discussions on the subject. We thank Michail Ryskin for an interesting correspondance and criticism. This research was supported in part by the Israel Science Foundation, founded by the Israeli Academy of Science and Humanities, by BSF grant \# 20004019 and by a grant from Israel Ministry of Science, Culture and Sport and the Foundation for Basic Research of the Russian Federation.

\section{References}

[1] P.D.B. Collins, “An introduction to Regge theory and high energy physics", Cambridge University Press 1977.

[2] Luca Caneschi (editor), "Regge Theory of Low - $p_{T}$ Hadronic Interaction", North-Holland 1989.

[3] E. Levin, "An introduction to pomerons," arXiv:hep-ph/9808486; "Everything about Reggeons. I: Reggeons in *soft* interaction," arXiv:hep-ph/9710546. 
[4] E. Gotsman, E. Levin and U. Maor, Phys. Lett. B452, (1999) 387; B309, 199 (1993); Phys. Rev. D49, (1994) R4321.

[5] V. A. Khoze, A. D. Martin and M. G. Ryskin, Eur. Phys. J. C18 (2000) 167; Phys. Lett. B643 (2006) 93.

A. B. Kaidalov, V. A. Khoze, A. D. Martin and M. G. Ryskin, Eur. Phys. J. C31 (2003) 387; C33 (2004) 261.

[6] E. Gotsman, E. Levin and U. Maor, "A Soft Interaction Model at Ultra High Energies: Amplitudes, Cross Sections and Survival ProEur. Phys. J. babilities," arXiv:0708.1506 [hep-ph].

[7] M. G. Ryskin, A. D. Martin and V. A. Khoze, Eur. Phys. J. C54 (2008) 199 [arXiv:0710.2494 [hep-ph]].

[8] V. N. Gribov, "Space-time description of hadron interactions at high energies," arXiv:hep-ph/0006158; Sov. J. Nucl. Phys. 9 (1969) 369 [ Yad. Fiz. 9 (1969) 640].

[9] A. Donnachie and P.V. Landshoff, Nucl. Phys. B231, (1984) 189; Phys. Lett. B296, (1992) 227; Zeit. Phys. C61, (1994) 139

[10] E. Gotsman, E. Levin and U. Maor, Phys. Rev. D60 (1999) 094011.

[11] E. Gotsman, E. Levin, U. Maor, E. Naftali and A. Prygarin, "HERA and the LHC - A workshop on the implications of HERA for LHC physics: Proceedings Part A" (2005) 221.

[12] E. Gotsman, A. Kormilitzin, E. Levin and U. Maor, Eur. Phys. J. C52 (2007) 295 [arXiv:hep-ph/0702053].

[13] G. P. Lepage and S. J. Brodsky, Phys. Rev. D 22 (1980) 2157.

[14] M. L. Good and W. D. Walker, Phys. Rev. 120 (1960) 1857.

[15] F. E. Low, Phys. Rev. D 12 (1975) 163; S. Nussinov, Phys. Rev. Lett. 34 (1975) 1286; E. A. Kuraev, L. N. Lipatov, and F. S. Fadin, Sov. Phys. JETP 45, 199 (1977); Ya. Ya. Balitsky and L. N. Lipatov, Sov. J. Nucl. Phys. 28, 22 (1978); A. H. Mueller, Nucl. Phys. B415, 373 (1994); B437, 107 (1995); L. V. Gribov, E. M. Levin and M. G. Ryskin, Phys. Rep. 100, 1 (1983); A. H. Mueller and J. Qiu, Nucl. Phys.,427 B 268 (1986) ; L. McLerran and R. Venugopalan, Phys. Rev. D 49,2233, 3352 (1994); D 50,2225 (1994); D 53,458 (1996); D 59,09400 (1999); L. N. Lipatov, Phys. Rept. 286, 131 (1997) [arXiv:hep-ph/9610276]; Sov. Phys. JETP 63, 904 (1986) and references therein.

[16] J. Bartels, M. Braun and G. P. Vacca, Eur. Phys. J. C40, 419 (2005) [arXiv:hep-ph/0412218]; J. Bartels and C. Ewerz, JHEP 9909, 026 (1999) [arXiv:hep-ph/9908454]; J. Bartels and M. Wusthoff, Z. Phys. C6Eur. Phys. J. 6, 157 (1995); $\quad$ A. H. Mueller and B. Patel, Nucl. Phys. B425, 471 (1994) [arXiv:hep-ph/9403256]; J. Bartels, Z. Phys. C60, 471 (1993).

[17] M. A. Braun, Phys. Lett. B632 (2006) 297 [arXiv:hep-ph/0512057]; Eur. Phys. J. C16, 337 (2000) [arXiv:hep-ph/0001268]; Phys. Lett. B 483 (2000) 115 [arXiv:hep-ph/0003004]; Eur. Phys. J. C 33 (2004) 113 [arXiv:hep-ph/0309293]; C6, 321 (1999) [arXiv:hep-ph/9706373]; M. A. Braun and G. P. Vacca, Eur. Phys. J. C6, 147 (1999) [arXiv:hep-ph/9711486].

[18] A. H. Mueller, Nucl. Phys. B 415 (1994) 373; B 437 (1995) 107.

[19] E. Laenen and E. Levin, Nucl. Phys. B 451 (1995) 207.

[20] P. Grassberger and K. Sundermeyer, Phys. Lett. B77, 220 (1978); E. Levin, Phys. Rev. D49, 4469 (1994); K. G. Boreskov, "Probabilistic model of Reggeon field theory," arXiv:hep-ph/0112325 and reference therein.

[21] E. Levin and M. Lublinsky, Nucl. Phys. A730, 191 (2004) [arXiv:hep-ph/0308279], A763,172 (2005), [arXiv:hep-ph/0501173]; Phys. Lett. B607, 131 (2005) [arXiv:hep-ph/0411121]; 
[22] Y. V. Kovchegov, Phys. Rev. D60, 034008 (1999), [arXiv:hep-ph/9901281].

[23] R. P. Feynman, Phys. Rev. Lett. 23 (1969) 1415; "Photon-Hadron Interactions," Reading 1972, 282p.

[24] M. Kozlov, E. Levin and A. Prygarin, Nucl. Phys. A 792 (2007) 122 [arXiv:0704.2124 [hep-ph]].

[25] S. Bondarenko, L. Motyka, A. H. Mueller, A. I. Shoshi and B. W. Xiao, Eur. Phys. J. C 50 (2007) 593 [arXiv:hep-ph/0609213].

[26] M. Kozlov and E. Levin, Nucl. Phys. A 779 (2006) 142 [ arXiv:hep-ph/0604039];

[27] D. Amati, M. Le Bellac, G. Marchesini and M.Ciafaloni, Nucl. Phys. B112 (1976) 107;

D. Amati, G. Marchesini, M.Ciafaloni and G. Parisi, Nucl. Phys. B114 (1976) 483.

[28] A. H. Mueller and B. Patel, Nucl. Phys. B425, 471 (1994); A. H. Mueller and G. P. Salam, Nucl. Phys. B475, 293 (1996), [arXiv:hep-ph/9605302]; G. P. Salam, Nucl. Phys. B461, 512 (1996); E. Iancu and A. H. Mueller, Nucl. Phys. A730 (2004) 460, 494, [arXiv:hep-ph/0308315],[arXiv:hep-ph/0309276].

[29] E. Levin and A. Prygarin, Eur. Phys. J. C 53 (2008) 385 [arXiv:hep-ph/0701178].

[30] E. Levin, J. Miller and A. Prygarin, "Summing Pomeron loops in the dipole approach,", Nucl.Phys.A (in press) arXiv:0706.2944 [hep-ph].

[31] M. Kozlov and E. Levin, Nucl. Phys. A739 (2004) 291 [arXiv:hep-ph/0401118].

[32] E. Levin, Nucl. Phys. A763, 140 (2005), [arXiv:hep-ph/0502243].

[33] Y. V. Kovchegov, Phys. Rev. D 72 (2005) 094009 [arXiv:hep-ph/0508276].

[34] M. Kozlov, E. Levin, V. Khachatryan and J. Miller, Nucl. Phys. A 791 (2007) 382 [arXiv:hep-ph/0610084].

[35] F. Abe et al., CDF collaboration, Phys. Rev. D 50 (1994) 5550.

[36] I. Gradstein and I. Ryzhik, "Tables of Series, Products, and Integrals”, Verlag MIR, Moskau,1981.

[37] V. A. Abramovsky, V. N. Gribov and O. V. Kancheli, Yad. Fiz. 18, 595 (1973) [ Sov. J. Nucl. Phys. 18, 308 (1974)].

[38] Y. V. Kovchegov and E. Levin, Nucl. Phys. B 577 (2000) 221 [arXiv:hep-ph/9911523].

[39] K. G. Boreskov, A. B. Kaidalov, V. A. Khoze, A. D. Martin and M. G. Ryskin, Eur. Phys. J. C 44 (2005) 523 [arXiv:hep-ph/0506211].

[40] ZEUS Collaboration, Nucl. Phys. B695 (2004) 3; Eur. Phys. J. C24 (2002) 345.

[41] K. Goulianos and J. Montanha, Phys. Rev.D59 (1999) 114017.

[42] F. Abe et al.(CDF Collaboration), Phys. Rev.D50 (1994) 5535.

[43] H.Kowalski and D. Teaney, Phys. Rev. D68 (2003) 114005.

[44] J. S. Miller, "Survival probability for Higgs diffractive production in high density QCD,", Eur. Phys. J. (in press), arXiv:hep-ph/0610427.

[45] V. A. Khoze, A. D. Martin and M. G. Ryskin, "New Physics with Tagged Forward Protons at the LHC," arXiv:0705.2314 [hep-ph] and references therein.

[46] J. Bartels, S. Bondarenko, K. Kutak and L. Motyka, Phys. Rev. D 73 (2006) 093004 [arXiv:hep-ph/0601128]. 